\title{
Study on the dynamic response of a curved railway track subjected to harmonic loads based on periodic structure theory
}

\author{
Weifeng Liu ${ }^{*}$, Linlin Du', Weining Liu', David J. Thompson² \\ 1. School of Civil Engineering, Beijing Jiaotong University, Beijing 100044, China.
}

2. Institute of Sound and Vibration Research, University of Southampton, Highfield, Southampton SO17 1BJ, UK

\section{Corresponding author}

Weifeng Liu, School of Civil Engineering, Beijing Jiaotong University, No.3 Shangyuancun, Haidian District, Beijing 100044, People's Republic of China.

Email: wfliu@bjtu.edu.cn

\begin{abstract}
The dynamic response of a curved railway track subjected to moving and non-moving harmonic loads is studied in this paper. The track is considered as a curved Timoshenko beam supported by periodically-spaced discrete fasteners. The displacement and rotation of the curved rail are expressed as the superposition of track modes in the frequency domain. Periodic structure theory is applied to the equations of motion of a curved track, allowing the dynamic response of the track to be calculated efficiently in a reference cell. The effect of the stiffness and damping of the fasteners, the fastener spacing and the radius of curvature on the mobility and decay rate of the track is analysed for non-moving loads on the rail head. The vibration of the rail under moving loads is also discussed. It is found that the dynamic response of a curved rail with a large radius has the same characteristics as that of a straight track. However, the dynamic response of the track is significantly affected when the radius of curvature becomes small. The radius affects the mobility, the decay rate below $2000 \mathrm{~Hz}$ and the velocity of the rail in the vertical direction when the radius is smaller than about $15 \mathrm{~m}$ and for the lateral direction when it is less than about $30 \mathrm{~m}$. Moreover, the curvature has a significant influence on the vertical/lateral cross mobility, the magnitude of which increases as the radius is reduced. When the radius is larger than $10 \mathrm{~m}$, the lateral vibration amplitude under a moving vertical load and the vertical response to a moving lateral load are inversely proportional to the radius.
\end{abstract}

Keywords: dynamic response; curved track; harmonic load; periodic structure theory; mode superposition 


\section{Introduction}

Vibration induced by railway or metro trains running in urban areas and transmitted through the ground can cause annoyance to residents, and may also affect historic buildings and sensitive instruments. The vibration levels are often higher in curved sections of line where the dynamic responses in the tunnel and on the ground surface are often greater than those for straight tracks ${ }^{1}$. The higher vibration levels are caused by the dynamic interaction of the train and the curved tracks. In the literature there are many models reported for the dynamic behaviour of railway tracks. Most of these focus on the response of straight tracks, whereas much less attention has been paid to curved tracks because of the higher complexity associated with the modelling of a curved track compared with a straight one.

The dynamic behaviour of rails is often studied by representing them as Euler-Bernoulli or Timoshenko beams. An Euler-Bernoulli beam has been found to be acceptable for frequencies below $500 \mathrm{~Hz}$ and a Timoshenko beam for frequencies up to at least $2 \mathrm{kHz}^{2,3}$. A model based on a curved beam is required for a curved track. The dynamic response of curved beams has been studied for many years. Both analytical methods and the finite element method have been employed in these previous studies. Generally, the finite element method is more applicable to studies of the dynamic response of curved beams with complicated structures than the analytical methods. The finite element method, however, remains an approximate approach, and its calculation results are affected by some factors such as the element types and the boundary conditions. Moreover, for a railway track, an infinite length should generally be considered, which is more difficult to implement in a finite element approach. Although it is sometimes difficult or even impossible to solve some problems using analytical methods, for example the response of a beam with an irregular cross-section, the analytical methods can provide more theoretical insight into the dynamic behaviour. In the analytical models, some assumptions are made to allow solution of the equations of motion. Provided that the beam cross-section is symmetric, the motion of a curved beam can be decoupled into in-plane (i.e. in the plane of curvature) and out-of-plane motions.

There have been a number of analytical studies on curved beams in recent years. For example, Yang et al. established a complete theory for treating the vibration of a horizontally curved Euler-Bernoulli beam subjected to a series of moving masses, each of which was simulated as a combination of a gravitational force and a centrifugal force ${ }^{4}$. Kang et al. provided a concise and efficient method for determining the free vibration of a multi-span circular curved beam with general boundary conditions and supports ${ }^{5}$. Yu et al. ${ }^{6}$ carried out an analytical study of the free vibration of a naturally curved and twisted beam with uniform cross-sectional shape using spatial 
curved beam theory based on Washizu's static model ${ }^{7}$. Çalım performed the forced vibration analysis of a curved beam on a two-parameter elastic foundation subjected to impulsive loads based on the Timoshenko beam theory ${ }^{8}$. Lee analyzed the in-plane free vibration of circularly curved Timoshenko beams through the pseudospectral method ${ }^{9}$. Howson and Jemah calculated the exact out-of-plane natural frequencies of curved Timoshenko beams by the dynamic stiffness method ${ }^{10}$.

Although the dynamic response of curved beams has been extensively studied, the application to curved railway tracks is limited, which is mainly attributed to the higher complexity of curved track models. Kostovasilis et al. established a finite element model of a curved track and compared the dynamic response obtained using straight beam elements and curved beam elements ${ }^{11}$. The curved beam model used in their study added complexity without giving substantial improvement for the specific application and therefore the straight element method was preferred. In another paper, Kostovasilis et al. used an analytical model to discuss the vertical/lateral coupling of the rail on a continuous elastic foundation including the effects of initial curvature ${ }^{12}$. In this model, the track is subjected to a non-moving harmonic load and the solution is obtained in the wavenumber domain using the Fourier transform method. Ang and Dai gave an analytical solution to the response of a curved railway track resting on a viscoelastic foundation subjected to a moving $\operatorname{load}^{13,14}$. In this work, trigonometric functions were employed as the trial functions to approximate the displacement of the curved rail. Li et al. performed an analytical study of the dynamic response of a curved track subjected to moving loads, and presented a model of a curved Timoshenko beam periodically supported by double-layer spring-damping elements ${ }^{15,16}$. In this model, the displacement of the rail under moving loads is expressed as the product of the load and the transfer function for the curved track based on the Duhamel integral and the dynamic reciprocity theorem. The transfer function was derived by using the transfer matrix method in the frequency domain. Zhang et al. discussed the dynamic response of a curved rail subjected to a moving train based on Li's research by coupling a two-dimensional vehicle model to the track model ${ }^{17}$.

Because most railway tracks are periodically supported by the sleepers, researchers have developed models of a straight track in which the periodicity of the track is exploited to increase the calculation efficiency. Some papers discuss the dynamic behaviour of track due to non-moving loads. Grassie et al. developed a model of a track represented as a periodically supported Timoshenko beam ${ }^{18}$. Gry and Gontier presented a periodic railway track model including cross-section deformation based on the notion of generalized cross-section displacements of a beam $^{19}$. In this model, the deformation of the beam's cross-section is described approximately in terms of cross-section modes, so the periodic analysis can be efficiently made using a small sized matrix equation. Sheng et al. gave a detailed discussion on the propagation and resonance properties 
of the track modelled as an infinitely long periodic Euler-Bernoulli beam ${ }^{20}$. Degrande et al. and Clouteau et al. presented a periodic track model as part of a periodic finite element-boundary element model based on the Floquet transform ${ }^{21,22}$. The dynamic response of track, tunnel and ground were calculated first in a reference cell, and then the response in other cells could be obtained through the inverse Floquet transformation.

The dynamic response of the track under moving loads has also been calculated through some models based on the periodicity of the track. Based on the work in [21, 22], Gupta et al. discussed the dynamic response of track, tunnel and ground due to moving loads ${ }^{23,24}$. Chebli et al. established a periodic track-ground model based on Floquet decomposition to predict the vibration of the track and ground ${ }^{25}$. Sheng et al. proposed a more general, wavenumber-based approach to study the response of an infinite periodic track under moving harmonic loads ${ }^{26}$. In this approach the periodically supported structure is represented as either a multiple-beam model or a two-and-half-dimensional finite-element model. Ma et al. considered a subway track as an infinite periodically supported Euler-Bernoulli beam and set up an analytical model; the vibration of a floating slab track and a general non-ballast track was calculated through this model ${ }^{27,28}$. In this model, the displacement of the track in the frequency domain was expressed as the superposition of track modes. In all the above studies, however, only straight tracks were considered.

This paper presents an analytical model of a curved, periodically supported track based on periodic structure theory. The periodicity of the track structure is applied to the equations of motion of a curved track to obtain efficiently the dynamic response in a reference cell of the track. The analytical solutions for a curved track under non-moving and moving harmonic loads are derived by separating the in-plane and out-of-plane motions. The effect of various parameters on the frequency response and decay rate of the track due to a non-moving load is analysed, and the vibration of the track under a moving load is also discussed.

\section{Dynamic response of a periodic curved track}

\subsection{Equations of motion of a curved track}

The curved rail is considered as a curved Timoshenko beam, as shown in Fig. 1, in which $\theta$ denotes the subtended angle, and $R$ is the radius of curvature. A right-handed coordinate system is used, the $x$ - and $y$-axis of which coincide with the principal axes of the cross-section, and the $z$-axis is tangential to the centroidal axis of the beam. $u_{x}, u_{y}$ and $u_{z}$ denote the displacements of the centroid of each cross-section of the curved beam along the three axes, and $\varphi_{x}, \varphi_{y}$ and $\varphi_{z}$ are the rotations 
about the three axes. All the deformations are assumed to be small so that linear theory applies. The curved beam is assumed to have a constant cross-section with negligible warping resistance.

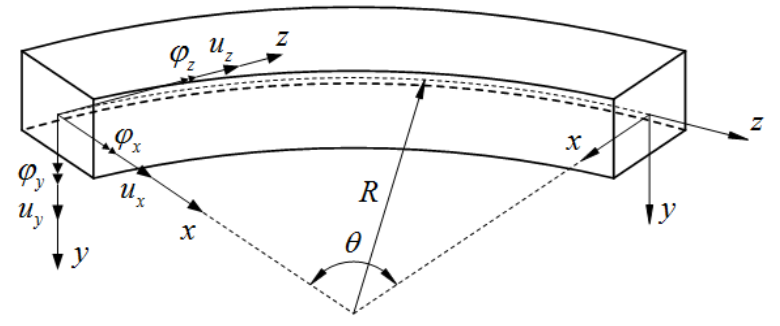

Fig. 1 Coordinates of curved beam

Let vertical and lateral harmonic forces $F_{v} \mathrm{e}^{\mathrm{i} \omega_{F} t}$ and $F_{l} \mathrm{e}^{\mathrm{i} \omega_{F} t}$, where $\omega_{F}$ is the excitation frequency, move along the rail head of the curved track at a speed $v$, as shown in Fig. 2. It should be noted that the vertical and lateral forces can be applied at any point on the rail head. The lateral force on the rail head is equivalent to a lateral force at the centroid and a moment $h_{1} \cdot F_{l} \mathrm{e}^{\mathrm{i} \omega_{F} t}$ acting about the $z$-axis, similarly a vertical force and a moment $b \cdot F_{v} \mathrm{e}^{\mathrm{i} \omega_{F} t}$ for the vertical force (see Fig. 3). The rail is supported by a finite number $N_{s}$ of periodically-spaced discrete fasteners. The fasteners are considered as springs and dashpots connected to the rail foot in the vertical, lateral and axial directions and torsional springs and dashpots connected at the shear centre of the rail.

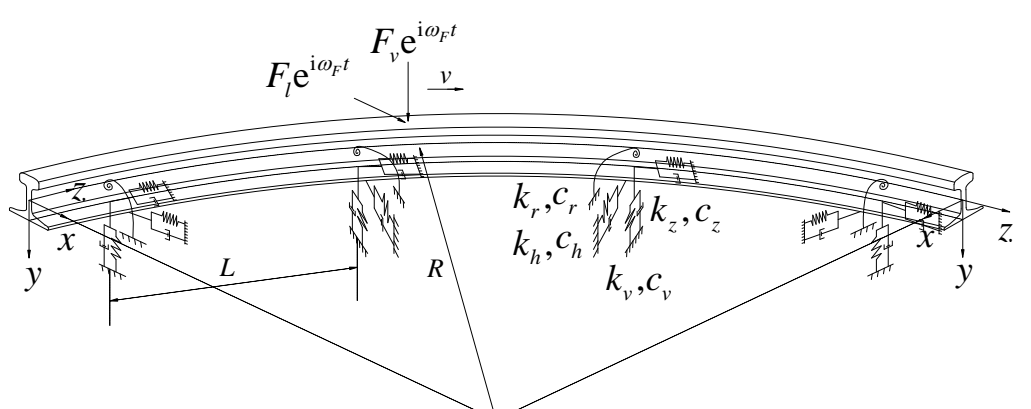

Fig. 2 Periodic curved track subjected to harmonic loads

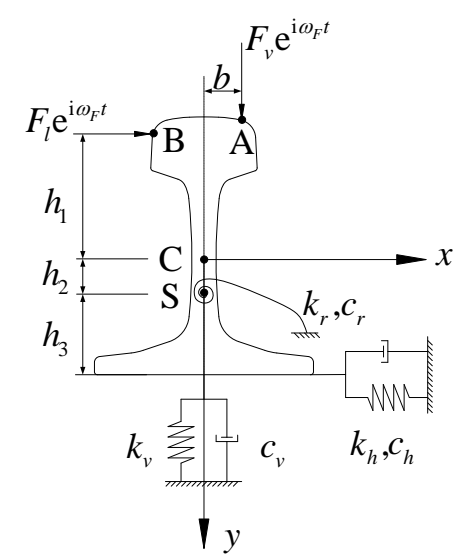

Fig. 3 Cross section of a rail ( $\mathrm{C}$ is the centroid, $\mathrm{S}$ the shear centre, $\mathrm{A}$ and $\mathrm{B}$ the force locations) 
According to the theory of curved Timoshenko beam, the equations of motion can be divided into those for the in-plane (lateral bending and axial) and the out-of-plane (vertical bending and torsional) motions. The equations for the in-plane motion of the curved track can be written as follows ${ }^{9,29}$.

$$
\begin{aligned}
& K_{x} A G\left(\frac{\partial u_{x}^{2}}{\partial z^{2}}-\frac{\partial \varphi_{y}}{\partial z}\right)+\frac{K_{x} A G+E A}{R} \frac{\partial u_{z}}{\partial z}-\frac{E A}{R^{2}} u_{x}-\rho A \frac{\partial^{2} u_{x}}{\partial t^{2}}+\sum_{j=1}^{N_{s}} f_{h j}(t) \delta\left(z-z_{s j}\right)=F_{l} \mathrm{e}^{\mathrm{i} \omega_{F} t} \delta\left(z-z_{0}^{F}-v t\right), \\
& E A \frac{\partial u_{z}^{2}}{\partial z^{2}}-\frac{K_{x} A G+E A}{R} \frac{\partial u_{x}}{\partial z}-\frac{K_{x} A G}{R}\left(\frac{u_{z}}{R}-\varphi_{y}\right)-\rho A \frac{\partial^{2} u_{z}}{\partial t^{2}}+\sum_{j=1}^{N_{s}} f_{z j}(t) \delta\left(z-z_{s j}\right)=0, \\
& E I_{y} \frac{\partial \varphi_{y}^{2}}{\partial z^{2}}+K_{x} A G\left(\frac{\partial u_{x}}{\partial z}+\frac{u_{z}}{R}-\varphi_{y}\right)-\rho I_{y} \frac{\partial^{2} \varphi_{y}}{\partial t^{2}}=0,
\end{aligned}
$$

where $E$ and $G$ denote the Young's modulus and shear modulus, respectively, of the rail, $A$ the cross-sectional area, $I_{y}$ the second moment of area about the $y$-axis, $K_{x}$ the cross-sectional shape factor about the $x$-axis, $\rho$ the density of the rail, $z_{0}^{F}$ the initial position of the moving load, and $z_{s j}$ the position of the $j^{\text {th }}$ fastener. The fasteners have been replaced by forces: $f_{h j}$ and $f_{z j}$ are the lateral and axial forces applied on the rail by the $j^{\text {th }}$ fastener. Equations (1), (2) and (3) correspond to the lateral displacement, the axial displacement along the $z$-axis and the rotation about the $y$-axis, respectively.

The equations for the out-of-plane motion of the curved track can be written as follows ${ }^{10,29}$.

$$
\begin{aligned}
& K_{y} A G \frac{\partial^{2} u_{y}}{\partial z^{2}}-K_{y} A G \frac{\partial \varphi_{x}}{\partial z}-\rho A \frac{\partial^{2} u_{y}}{\partial t^{2}}+\sum_{j=1}^{N_{s}} f_{v j}(t) \delta\left(z-z_{s j}\right)=F_{v} \mathrm{e}^{\mathrm{i} \omega_{F} t} \delta\left(z-z_{0}^{F}-v t\right), \\
& E I_{x} \frac{\partial^{2} \varphi_{x}}{\partial z^{2}}-\frac{E I_{x}+G I_{d}}{R} \frac{\partial \varphi_{z}}{\partial z}+K_{y} A G \frac{\partial u_{y}}{\partial z}-\left(\frac{G I_{d}}{R^{2}}+K_{y} A G\right) \varphi_{x}-\rho I_{x} \frac{\partial^{2} \varphi_{x}}{\partial t^{2}}=0, \\
& G I_{d} \frac{\partial^{2} \varphi_{z}}{\partial z^{2}}+\frac{E I_{x}+G I_{d}}{R} \frac{\partial \varphi_{x}}{\partial z}-\frac{E I_{x}}{R^{2}} \varphi_{z}-\rho I_{0} \frac{\partial^{2} \varphi_{z}}{\partial t^{2}}+\sum_{j=1}^{N_{s}} T_{j}(t) \delta\left(z-z_{s j}\right)=\left(h_{1} F_{l} \mathrm{e}^{\mathrm{i} \omega_{F} t}+b F_{v} \mathrm{e}^{\mathrm{i} \omega_{F} t}\right) \delta\left(z-z_{0}^{F}-v t\right),
\end{aligned}
$$

where $I_{x}$ denotes the second moment of area about the $x$-axis, $I_{0}$ the polar moment of area, $I_{d}$ the torsional constant, $K_{y}$ the cross-sectional shape factor about $y$-axis, $f_{v j}$ and $T_{j}$ the vertical force and torsional moment applied on the rail by the $j^{\text {th }}$ fastener respectively. Equations (4), (5) and (6) correspond to the vertical displacement, and the rotations about the $x$-axis and $z$-axis, respectively.

Equations (1)-(3) for the in-plane displacements and rotation of the curved track are independent of equations (4)-(6) for the out-of-plane displacement and rotations apart from the forcing terms, so the in-plane and out-of-plane dynamic responses can be determined separately. 


\subsection{Transformation to the frequency domain}

Based on the Fourier transformation, the frequency spectrum of a dynamic response can be analysed. Applying the Fourier transformation with respect to time to equations (1)-(3), and introducing the support stiffnesses, the in-plane equations can be obtained in the frequency domain:

$$
\begin{aligned}
& K_{x} A G^{*}\left(\frac{\partial \hat{u}_{x}^{2}}{\partial z^{2}}-\frac{\partial \hat{\varphi}_{y}}{\partial z}\right)+\frac{K_{x} A G^{*}+E^{*} A}{R} \frac{\partial \hat{u}_{z}}{\partial z}+\left(\rho A \omega^{2}-\frac{E^{*} A}{R^{2}}\right) \hat{u}_{x}+\bar{k}_{h} \sum_{j=1}^{N_{s}} \hat{u}_{x} \delta\left(z-z_{s j}\right)=\frac{1}{v} F_{l} \mathrm{e}^{\mathrm{i} \frac{\omega_{F}-\omega}{v}\left(z-z_{0}^{F}\right)}, \\
& E^{*} A \frac{\partial \hat{u}_{z}^{2}}{\partial z^{2}}-\frac{K_{x} A G^{*}+E^{*} A}{R} \frac{\partial \hat{u}_{x}}{\partial z}+\frac{K_{x} A G^{*}}{R} \hat{\varphi}_{y}+\left(\rho A \omega^{2}-\frac{K_{x} A G^{*}}{R^{2}}\right) \hat{u}_{z}+\bar{k}_{z} \sum_{j=1}^{N_{s}} \hat{u}_{z} \delta\left(z-z_{s j}\right)=0, \\
& E^{*} I_{y} \frac{\partial \hat{\varphi}_{y}^{2}}{\partial z^{2}}+K_{x} A G^{*}\left(\frac{\partial \hat{u}_{x}}{\partial z}+\frac{\hat{u}_{z}}{R}\right)+\left(\rho I_{y} \omega^{2}-K_{x} A G^{*}\right) \hat{\varphi}_{y}=0,
\end{aligned}
$$

where “ $\wedge$ " is used to indicate expressions in the frequency domain; damping is introduced by making $E^{*}=E(1+\mathrm{i} \eta)$ and $G^{*}=G(1+\mathrm{i} \eta)$ complex, in which $\eta$ is the damping loss factor of the rail; the lateral forces applied by the fasteners have been replaced by introducing the dynamic stiffnesses $\bar{k}_{h}=k_{h}+\mathrm{i} c_{h} \omega$ and $\bar{k}_{z}=k_{z}+\mathrm{i} c_{z} \omega$, in which $k_{h}$ and $k_{z}$ are the lateral and axial stiffnesses of the fastener, and $c_{h}$ and $c_{z}$ are the corresponding lateral and axial damping coefficients.

Similarly the out-of-plane equations in the frequency domain are obtained as follows.

$$
\begin{aligned}
& K_{y} A G^{*} \frac{\partial^{2} \hat{u}_{y}}{\partial z^{2}}-K_{y} A G^{*} \frac{\partial \hat{\varphi}_{x}}{\partial z}+\rho A \omega^{2} \hat{u}_{y}+\bar{k}_{v} \sum_{j=1}^{N_{s}} \hat{u}_{y} \delta\left(z-z_{s j}\right)=\frac{1}{v} F_{v} \mathrm{e}^{\mathrm{i} \frac{\omega_{F}-\omega}{v}\left(z-z_{0}^{F}\right)}, \\
& E^{*} I_{x} \frac{\partial^{2} \hat{\varphi}_{x}}{\partial z^{2}}-\frac{E^{*} I_{x}+G^{*} I_{d}}{R} \frac{\partial \hat{\varphi}_{z}}{\partial z}+K_{y} A G^{*} \frac{\partial \hat{u}_{y}}{\partial z}-\left(\frac{G^{*} I_{d}}{R^{2}}+K_{y} A G^{*}+\rho I_{x} \omega^{2}\right) \hat{\varphi}_{x}=0, \\
& G^{*} I_{d} \frac{\partial^{2} \hat{\varphi}_{z}}{\partial z^{2}}+\frac{E^{*} I_{x}+G^{*} I_{d}}{R} \frac{\partial \hat{\varphi}_{x}}{\partial z}+\left(\rho I_{0} \omega^{2}-\frac{E^{*} I_{x}}{R^{2}}\right) \hat{\varphi}_{z}+\bar{k}_{r} \sum_{j=1}^{N_{s}} \hat{\varphi}_{z} \delta\left(z-z_{s j}\right)=\frac{h_{1} F_{l}+b F_{v}}{v} \mathrm{e}^{\mathrm{i} \frac{\omega_{F}-\omega}{v}\left(z-z_{0}^{F}\right)},
\end{aligned}
$$

where the vertical and rotational forces applied by the fasteners have been replaced by introducing the dynamic stiffnesses $\bar{k}_{v}=k_{v}+\mathrm{i} c_{v} \omega, \bar{k}_{r}=k_{r}+\mathrm{i} c_{r} \omega$, in which $k_{v}$ and $k_{r}$ are the vertical and torsional stiffnesses of the fastener, and $c_{v}$ and $c_{r}$ are the vertical and torsional damping coefficients of the fastener.

If the curved rail is supported by a continuous elastic foundation, the response of the rail can also be calculated. The forces applied on the rail by the fasteners in equations (7)-(12) can be replaced with product of the dynamic stiffness of the foundation and the displacement of the rail, 
for example $\bar{k}_{h}^{f} \hat{u}_{x}$ in equation (7), where $\bar{k}_{h}^{f}$ is the lateral dynamic stiffness of the foundation per unit length. In this calculation, the stiffness and damping of the foundation per unit length are equal to those of the fastener divided by the fastener spacing.

\subsection{Periodic structure theory and mode function of the track}

In this subsection, the periodic structure theory and the mode function of the track are introduced to solve the above equations of motion.

Based on the periodicity of the track structure along the $z$-axis, periodic structure theory can be used. Assume that the infinitely long track is composed of cells, each with the same properties, and any cell can be chosen as the reference cell. The rail displacements due to a moving harmonic unit load in another cell can be linked to those in the reference cell ${ }^{30,31}$,

$\hat{\mathbf{u}}\left(\bar{z}+n_{c} L, \omega, \omega_{F}\right)=\mathrm{e}^{\mathrm{i}\left(\omega_{F}-\omega\right) n_{c} L / v} \hat{\mathbf{u}}\left(\bar{z}, \omega, \omega_{F}\right)$,

where " $\sim$ " is used to indicate the expression in the reference cell, $\mathbf{u}=\left(u_{x}, \varphi_{y}, u_{z}, \varphi_{x}, u_{y}, \varphi_{z}\right)^{T}, L$ is the length of each cell, and $n_{c}$ is the index of the cells. A function $\hat{\mathbf{P}}\left(\bar{z}, \omega, \omega_{F}\right)$ is defined here:

$\hat{\mathbf{P}}\left(\bar{z}, \omega, \omega_{F}\right)=\hat{\mathbf{u}}\left(\bar{z}, \omega, \omega_{F}\right) \mathrm{e}^{-\mathrm{i}\left(\omega_{F} / v-\omega / v\right) \bar{z}}$.

Substituting equation (13) into equation (14), we can obtain:

$\hat{\mathbf{P}}\left(\widehat{z}+n_{c} L, \omega, \omega_{F}\right)=\hat{\hat{\mathbf{P}}}\left(\widehat{z}, \omega, \omega_{F}\right)$.

Because $\hat{\mathbf{P}}\left(\bar{z}, \omega, \omega_{F}\right)$ is a periodic function, it can be decomposed as a Fourier series.

$\hat{\mathbf{P}}\left(\bar{z}, \omega, \omega_{F}\right)=\sum_{n=-\infty}^{+\infty} \mathbf{C}_{n}\left(\omega, \omega_{F}\right) \mathrm{e}^{\mathrm{i} \xi_{n} \bar{z}}$,

where $\xi_{n}=2 \pi n / L$, and $\mathbf{C}=\left(U_{x}, \Phi_{y}, U_{z}, \Phi_{x}, U_{y}, \Phi_{z}\right)^{T}$ is a vector of coefficients.

Specifically, the displacements of the rail can be written as

$\hat{\mathbf{u}}\left(\bar{z}, \omega, \omega_{F}\right)=\sum_{n=-\infty}^{+\infty} \mathbf{C}_{n}\left(\omega, \omega_{F}\right) \hat{\bar{V}}_{n}\left(\widehat{z}, \omega, \omega_{F}\right)$.

where $\hat{V}_{n}\left(\bar{z}, \omega, \omega_{F}\right)=\mathrm{e}^{\mathrm{i}\left(\xi_{n}+\omega_{F} / v-\omega / v\right) \bar{z}}$, which can be called the mode function of the track.

In the calculation, $2 N+1$ modes can be considered, so the displacements are written as

$\hat{\mathbf{\mathbf { u }}}\left(\widehat{z}, \omega, \omega_{F}\right) \approx \sum_{n=-N}^{+N} \mathbf{C}_{n}\left(\omega, \omega_{F}\right) \hat{\hat{V}}_{n}\left(\bar{z}, \omega, \omega_{F}\right)$.

$N$ should be enough large to ensure the accuracy of the calculation.

Based on equation (13), the following boundary conditions of the reference cell should be satisfied.

$\left.\hat{\mathbf{F}}_{i n}\right|_{z=L}=\left.\mathrm{e}^{\mathrm{i}\left(\omega_{F}-\omega\right) L / v} \hat{\overline{\mathbf{F}}}_{i n}\right|_{z=0}$ 
where $\mathbf{F}_{\text {in }}=\left(Q_{x}, M_{y}, N_{z}, M_{x}, Q_{y}, T_{z}\right)^{T}$ represents the internal force vector including the shear force $Q_{x}$, the bending moment $M_{y}$ and the axial force $N_{z}$, which are the in-plane terms, and the bending moment $M_{x}$, the shear force $Q_{y}$ and the twisting moment $T_{z}$, which are the out-of-plane terms. According to the theory of a curved Timoshenko beam, these internal forces can be written as ${ }^{9,32}$

$$
\begin{aligned}
& \left\{\begin{array}{l}
\hat{Q}_{x}(z, \omega)=K_{x} A G\left(\frac{\hat{u}_{z}(z, \omega)}{R}+\frac{\partial \hat{u}_{x}(z, \omega)}{\partial z}-\hat{\varphi}_{y}(z, \omega)\right) \\
\hat{M}_{y}(z, \omega)=E I_{y} \frac{\partial \hat{\varphi}_{y}(z, \omega)}{\partial z}, \\
\hat{N}_{z}(z, \omega)=E A\left(\frac{\partial \hat{u}_{z}(z, \omega)}{\partial z}-\frac{\hat{u}_{x}(z, \omega)}{R}\right)
\end{array}\right. \\
& \left\{\begin{array}{l}
\hat{M}_{x}(z, \omega)=-E I_{x}\left(\frac{\partial \hat{\varphi}_{x}(z, \omega)}{\partial z}-\frac{\hat{\varphi}_{z}(z, \omega)}{R}\right) \\
\hat{Q}_{y}(z, \omega)=K_{y} A G\left(\frac{\partial \hat{u}_{y}(z, \omega)}{\partial z}-\hat{\varphi}_{x}(z, \omega)\right) \\
\hat{T}_{z}(z, \omega)=G I_{d}\left(\frac{\hat{\varphi}_{x}(z, \omega)}{R}+\frac{\partial \hat{\varphi}_{z}(z, \omega)}{\partial z}\right) .
\end{array}\right.
\end{aligned}
$$

Substituting equation (18) into equations (21) and (22), it can be found that equations (19) and (20) are easily satisfied for each value of $n$ and hence for the overall response.

\subsection{Solution to equations of motion}

Fig. 4 shows the solution process diagrammatically. The specific vectors and matrices in Fig. 4 will be explained in the following derivation. 


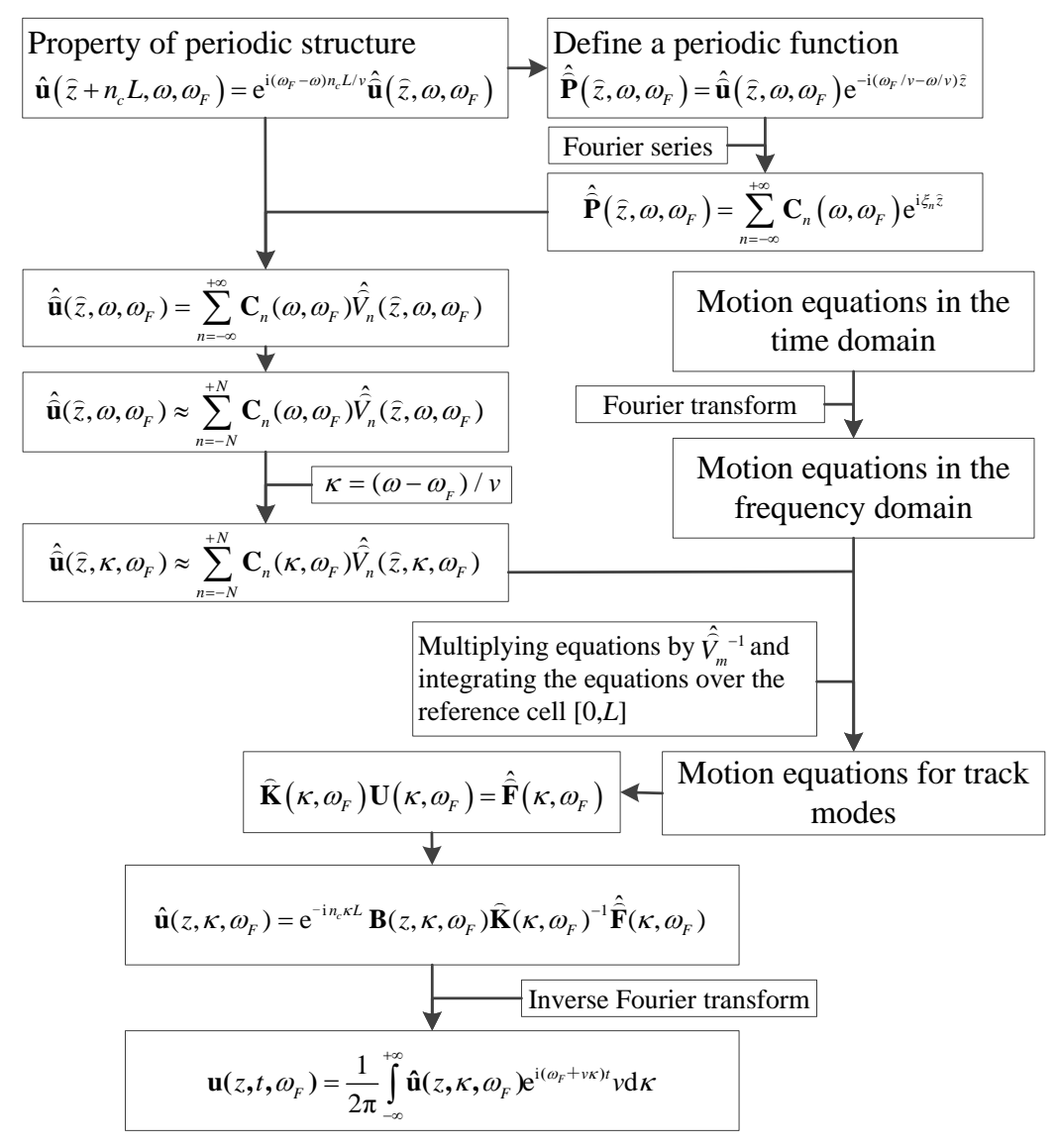

Fig. 4 Solution process of equations of motion of a curved track

Writing $\kappa=\left(\omega-\omega_{F}\right) / v$, the displacements of the rail from equation (17) can also be written as $\hat{\mathbf{\mathbf { u }}}\left(\widehat{z}, \kappa, \omega_{F}\right) \approx \sum_{n=-N}^{+N} \mathbf{C}_{n}\left(\kappa, \omega_{F}\right) \hat{\hat{V}}_{n}\left(\widehat{z}, \kappa, \omega_{F}\right)$.

Multiplying both sides of the in-plane equations (7)-(9) by $\hat{\bar{V}}_{m}\left(\bar{z}, \kappa, \omega_{F}\right)^{-1}=\mathrm{e}^{-\mathrm{i}\left(\xi_{m}-\kappa\right) \bar{z}}(m \in[-N,+N])$, and integrating the equations over the length $[0, L]$ of the reference cell, we can obtain:

$$
\begin{aligned}
& {\left[-K_{x} A G^{*}\left(\xi_{m}-\kappa\right)^{2}+\rho A\left(\omega_{F}+v \kappa\right)^{2}-\frac{E^{*} A}{R^{2}}\right] L U_{x m}-\mathrm{i} K_{x} A G^{*}\left(\xi_{m}-\kappa\right) L \Phi_{y m}+\mathrm{i} \frac{K_{x} A G^{*}+E^{*} A}{R}\left(\xi_{m}-\kappa\right) L U_{z m}} \\
& +\bar{k}_{h} \sum_{j=1}^{N_{s}} \hat{\bar{V}}_{m}\left(\bar{z}_{r j}, \kappa, \omega_{F}\right)^{-1} \hat{\vec{u}}_{x}=\frac{1}{v} \cdot F_{l} \mathrm{e}^{\mathrm{i} \kappa \hat{z}_{0}^{F}} \int_{0}^{L} \mathrm{e}^{-\mathrm{i} \xi_{m} \hat{z}} d \bar{z} \\
& {\left[\rho A\left(\omega_{F}+v \kappa\right)^{2}-K_{x} A G^{*}-E^{*} A\left(\xi_{m}-\kappa\right)^{2}\right] L U_{z m}-\mathrm{i} \frac{K_{x} A G^{*}+E^{*} A}{R}\left(\xi_{m}-\kappa\right) L U_{x m}+\frac{K_{x} A G^{*}}{R} L \Phi_{y m},} \\
& +\bar{k}_{z} \sum_{j=1}^{N_{s}} \hat{\hat{V}}_{m}\left(\bar{z}_{r j}, \kappa, \omega_{F}\right)^{-1} \hat{\vec{u}}_{z}=0 \\
& {\left[\rho I_{y}\left(\omega_{F}+v \kappa\right)^{2}-K_{x} A G^{*}-E^{*} I_{y}\left(\xi_{m}-\kappa\right)^{2}\right] L \Phi_{y m}+\mathrm{i} K_{x} A G^{*}\left(\xi_{m}-\kappa\right) L U_{x m}+\frac{K_{x} A G^{*}}{R} L \Phi_{z m}=0,}
\end{aligned}
$$

Similarly, the out-of-plane equations become: 
$\left[-K_{y} A G^{*}\left(\xi_{m}-\kappa\right)^{2}+\rho A\left(\omega_{F}+v \kappa\right)^{2}\right] L U_{y m}-\mathrm{i} K_{y} A G^{*}\left(\xi_{m}-\kappa\right) L \Phi_{x m}$

$+\bar{k}_{v} \sum_{j=1}^{N_{s}} \hat{\hat{V}}_{m}\left(\widehat{z}_{r j}, \kappa, \omega_{F}\right)^{-1} \hat{\vec{u}}_{y}=\frac{1}{v} \cdot F_{v} \mathrm{e}^{\mathrm{i} \kappa \hat{z}_{0}^{F}} \int_{0}^{L} \mathrm{e}^{-\mathrm{i} \xi_{m} \bar{z}} d \bar{z}$

$\left[\frac{G^{*} I_{d}}{R^{2}}+K_{y} A G^{*}+\rho I_{x}\left(\omega_{F}+v \kappa\right)^{2}+E^{*} I_{x}\left(\xi_{m}-\kappa\right)^{2}\right] L \Phi_{x m}+\mathrm{i} \frac{E^{*} I_{x}+G^{*} I_{d}}{R}\left(\xi_{m}-\kappa\right) L \Phi_{z m}$,

$-\mathrm{i} K_{y} A G^{*}\left(\xi_{m}-\kappa\right) L U_{y m}=0$

$\left[\rho I_{0} \omega^{2}-\frac{E^{*} I_{x}}{R^{2}}-G^{*} I_{d}\left(\xi_{m}-\kappa\right)^{2}\right] L \Phi_{z m}-\mathrm{i} \frac{E^{*} I_{x}+G^{*} I_{d}}{R}\left(\xi_{m}-\kappa\right) L \Phi_{x m}+\bar{k}_{r} \sum_{j=1}^{N_{s}} \hat{\hat{V}}_{m}\left(\hat{z}_{r j}, \kappa, \omega_{F}\right)^{-1} \hat{\bar{\varphi}}_{z}$

$=\frac{h_{1} F_{l}+b F_{v}}{v} \cdot \mathrm{e}^{\mathrm{i} \kappa \hat{z}_{0}^{F}} \int_{0}^{L} \mathrm{e}^{-\mathrm{i} \xi_{m} \hat{z}} d \bar{z}$

In equations (24), (27) and (29), the integral on the right-hand side can be evaluated as

$\frac{1}{v} \cdot F_{l} \mathbf{e}^{\mathbf{i} \kappa z_{0}^{F}} \int_{0}^{L} \mathbf{e}^{-\mathbf{i} \xi_{m} \bar{z}} d \bar{z}=\left\{\begin{array}{c}\frac{L}{v} \cdot F_{l} \mathbf{e}^{\mathbf{i} \kappa_{z_{0}^{F}}^{F}},(m=0) . \\ 0, \quad(m \neq 0)\end{array}\right.$

Because $m \in[-N,+N]$, in equation (24)

$$
\begin{aligned}
& \bar{k}_{h} \sum_{j=1}^{N_{s}} \hat{\hat{V}}_{m}\left(\widehat{z}_{r j}, \kappa, \omega_{F}\right)^{-1} \hat{\vec{u}}_{x}=
\end{aligned}
$$

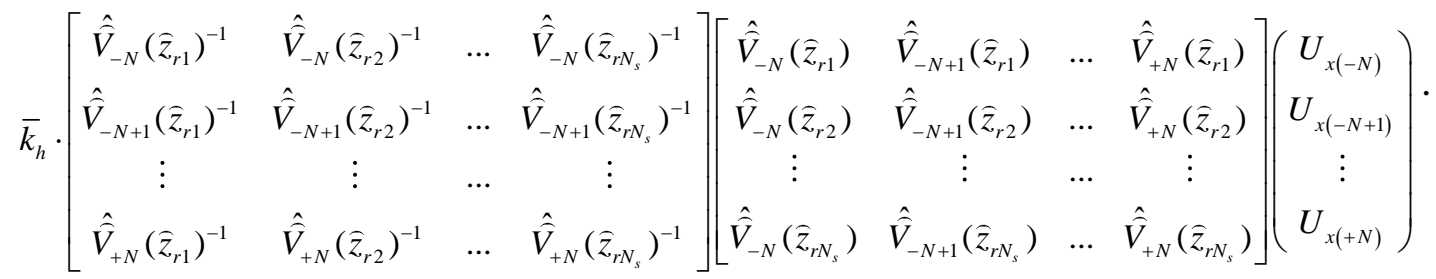

A similar expression can be obtained for the terms related to the fastener in equations (25), (27) and (29). The in-plane equations (24)-(26) can be written as

$\widehat{\mathbf{K}}_{i n}\left(\kappa, \omega_{F}\right) \mathbf{U}_{i n}\left(\kappa, \omega_{F}\right)=\hat{\hat{\mathbf{F}}}_{i n}\left(\kappa, \omega_{F}\right)$

where $\mathbf{U}_{i n}\left(\kappa, \omega_{F}\right)=\left\{U_{x(-N)}, \cdots, U_{x(+N)}, \Phi_{y(-N)}, \cdots, \Phi_{y(+N)}, U_{z(-N)}, \cdots, U_{z(+N)}\right\}^{\mathrm{T}} ; \quad \widehat{\mathbf{K}}_{i n}\left(\kappa, \omega_{F}\right)$ is the generalized stiffness matrix; $\hat{\mathbf{F}}_{i n}\left(\kappa, \omega_{F}\right)$ is the force vector, which can also be written as

$\hat{\mathbf{F}}_{i n}\left(\kappa, \omega_{F}\right)=\mathbf{F}_{i n}^{\prime} L \mathrm{e}^{\mathrm{i} \kappa z_{0}^{F}} / v$,

where the $j^{\text {th }}$ element of $\mathbf{F}_{i n}^{\prime}$ is

$F_{i n(j)}^{\prime}=\left\{\begin{array}{ll}F_{l}, & (j=N+1) \\ 0, & (j=\text { others })\end{array}\right.$.

Similarly, the out-of-plane equations (27)-(29) can be written as

$\widehat{\mathbf{K}}_{\text {out }}\left(\kappa, \omega_{F}\right) \mathbf{U}_{\text {out }}\left(\kappa, \omega_{F}\right)=\hat{\mathbf{F}}_{\text {out }}\left(\kappa, \omega_{F}\right)$,

where $\mathbf{U}_{\text {out }}\left(\kappa, \omega_{F}\right)=\left\{\Phi_{x(-N)}, \cdots, \Phi_{x(+N)}, U_{y(-N)}, \cdots, U_{y(+N)}, \Phi_{z(-N)}, \cdots, \Phi_{z(+N)}\right\}^{\mathrm{T}}$. 
$\hat{\mathbf{F}}_{\text {out }}\left(\kappa, \omega_{F}\right)=\mathbf{F}_{\text {out }}^{\prime} L \mathrm{e}^{\mathrm{i} \kappa \varkappa_{0}^{F}} / v$,

where the $j^{\text {th }}$ element of $\mathbf{F}_{\text {out }}^{\prime}$ is

$$
F_{\text {out }(j)}^{\prime}=\left\{\begin{array}{cc}
F_{v}, & (j=N+1) \\
h_{1} F_{l}+b F_{v}, & (j=5 N+3) . \\
0, & (j=\text { others })
\end{array}\right.
$$

The frequency responses of the rail at any point can be obtained:

$$
\begin{aligned}
& \hat{\mathbf{u}}_{\text {in }}\left(z, \kappa, \omega_{F}\right)=\mathrm{e}^{-\mathrm{i} n_{c} \kappa L} \mathbf{B}\left(z, \kappa, \omega_{F}\right) \widehat{\mathbf{K}}_{\text {in }}\left(\kappa, \omega_{F}\right)^{-1} \hat{\mathbf{F}}_{\text {in }}\left(\kappa, \omega_{F}\right), \\
& \hat{\mathbf{u}}_{\text {out }}\left(z, \kappa, \omega_{F}\right)=\mathrm{e}^{-\mathrm{i} n_{c} \kappa L} \mathbf{B}\left(z, \kappa, \omega_{F}\right) \widehat{\mathbf{K}}_{\text {out }}\left(\kappa, \omega_{F}\right)^{-1} \hat{\overline{\mathbf{F}}}_{\text {out }}\left(\kappa, \omega_{F}\right),
\end{aligned}
$$

where $\mathbf{B}\left(z, \kappa, \omega_{F}\right)$ is the mode matrix, given by

$$
\mathbf{B}\left(z, \kappa, \omega_{F}\right)=\left[\begin{array}{ccccccccc}
\mathrm{e}^{\mathrm{i}\left(\xi_{-N}-\kappa\right) z} & \cdots & \mathrm{e}^{\mathrm{i}\left(\xi_{+N}-\kappa\right) z} & 0 & \cdots & 0 & 0 & \cdots & 0 \\
0 & \cdots & 0 & \mathrm{e}^{\mathrm{i}\left(\xi_{-N}-\kappa\right) z} & \cdots & \mathrm{e}^{\mathrm{i}\left(\xi_{+N}-\kappa\right) z} & 0 & \cdots & 0 \\
0 & \cdots & 0 & 0 & \cdots & 0 & \mathrm{e}^{\mathrm{i}\left(\xi_{-N}-\kappa\right) z} & \cdots & \mathrm{e}^{\mathrm{i}\left(\xi_{+}-\kappa\right) z}
\end{array}\right] .
$$

Through the inverse Fourier transformation, the dynamic responses of the rail in the time domain due to a moving harmonic force can be obtained as:

$$
\begin{aligned}
& \mathbf{u}\left(z, t, \omega_{F}\right)=\frac{1}{2 \pi} \int_{-\infty}^{+\infty} \hat{\mathbf{u}}\left(z, \omega, \omega_{F}\right) \mathrm{e}^{\mathrm{i} \omega t} \mathrm{~d} \omega=\frac{1}{2 \pi} \int_{-\infty}^{+\infty} \hat{\mathbf{u}}\left(z, \kappa, \omega_{F}\right) \mathrm{e}^{\mathrm{i}\left(\omega_{F}+v \kappa\right) t} v \mathrm{~d} \kappa \\
& =\left[\frac{1}{2 \pi} \int_{-\infty}^{+\infty} L \mathrm{e}^{-\mathrm{i} \kappa\left(n_{c} L-z_{0}^{F}\right)} \mathbf{B}\left(z, \kappa, \omega_{F}\right) \hat{\mathbf{K}}\left(\kappa, \omega_{F}\right)^{-1} \mathbf{F}^{\prime} \mathrm{e}^{\mathrm{i} v \kappa t} \mathrm{~d} \kappa\right] \mathrm{e}^{\mathrm{i} \omega_{F} t}
\end{aligned}
$$

where $\hat{\mathbf{K}}\left(\kappa, \omega_{F}\right)$ can be $\hat{\mathbf{K}}_{\text {in }}\left(\kappa, \omega_{F}\right)$ or $\widehat{\mathbf{K}}_{\text {out }}\left(\kappa, \omega_{F}\right)$, and $\mathbf{F}^{\prime}$ can be $\mathbf{F}_{\text {in }}^{\prime}$ or $\mathbf{F}_{\text {out }}^{\prime}$.

Equation (41) gives the dynamic response of the rail subjected to the moving harmonic load. If we set $v=0$, the response of the rail due to a non-moving harmonic load applied at $z=0$ can be expressed as

$$
\mathbf{u}\left(z, t, \omega_{F}\right)=\left[\frac{1}{2 \pi} \int_{-\infty}^{+\infty} L \mathrm{e}^{-\mathrm{i} \kappa\left(n_{c} L-z_{0}^{F}\right)} \mathbf{B}\left(z, \kappa, \omega_{F}\right) \widehat{\mathbf{K}}\left(\kappa, \omega_{F}\right)^{-1} \mathbf{F}^{\prime} \mathrm{d} \kappa\right] \mathrm{e}^{\mathrm{i} \omega_{F} t}
$$

From equation (42), the amplitude of the harmonic response of the rail can be calculated by numerical integration:

$\overline{\mathbf{u}}\left(z, \omega_{F}\right) \approx \frac{1}{2 \pi} \sum_{j=1}^{M} L \mathrm{e}^{-\mathrm{i} \kappa_{j}\left(n_{c} L-z_{0}^{F}\right)} a_{j} \mathbf{B}\left(z, \kappa_{j}, \omega_{F}\right) \widehat{\mathbf{K}}\left(\kappa_{j}, \omega_{F}\right)^{-1} \mathbf{F}^{\prime} \Delta \kappa$

where $a_{1}=0.5, a_{M}=0.5$ and $a_{j}=1(j \in(1, M)) . \Delta \kappa$ should be sufficiently small to ensure the required accuracy. 


\section{Model validation}

To verify the validity of the analytical model described above, two comparisons are made between this model and solutions provided in the literature, [11] and [13].

In [11], the vertical displacement of the rail due to a moving load on a curved track was calculated using a finite element model. The parameters of this calculation are shown in Table 1.

Table 1 Parameters of calculation from [11]

\begin{tabular}{ll}
\hline Young's modulus of rail $E$ & $2.1 \times 10^{11} \mathrm{~N} / \mathrm{m}^{2}$ \\
Shear modulus of rail $G$ & $7.956 \times 10^{10} \mathrm{~N} / \mathrm{m}^{2}$ \\
Cross-sectional area $A$ & $7.672 \times 10^{-3} \mathrm{~m}^{2}$ \\
Second moment of area of rail for vertical bending $I_{x}$ & $3.038 \times 10^{-5} \mathrm{~m}^{4}$ \\
Second moment of area of rail for lateral bending $I_{y}$ & $0.5123 \times 10^{-5} \mathrm{~m}^{4}$ \\
Polar moment of inertia of rail $I_{0}$ & $3.551 \times 10^{-5} \mathrm{~m}^{4}$ \\
Damping loss factor of rail $\eta$ & 0.1 \\
Density of rail $\rho$ & $7850 \mathrm{~kg} / \mathrm{m}^{3}$ \\
Vertical stiffness of fastener $k_{v}$ & $20 \mathrm{MN} / \mathrm{m}^{2}$ \\
Fastener spacing $L$ & $0.6 \mathrm{~m}$ \\
Radius of curvature $R$ & $20 \mathrm{~m}$ \\
Speed of load $v$ & $20 \mathrm{~m} / \mathrm{s}$ \\
Load magnitude $F_{v}$ & $1 \mathrm{~N}$ \\
\hline
\end{tabular}

The results are compared with those from [11] in Fig. 5. This shows the displacement at mid-span between two fasteners due to a harmonic moving load in two excitation frequency cases of $0 \mathrm{~Hz}$ and $20 \mathrm{~Hz}$. It can be seen that the results calculated by the present model show a good agreement with the numerical solutions from [11]. 
(a)

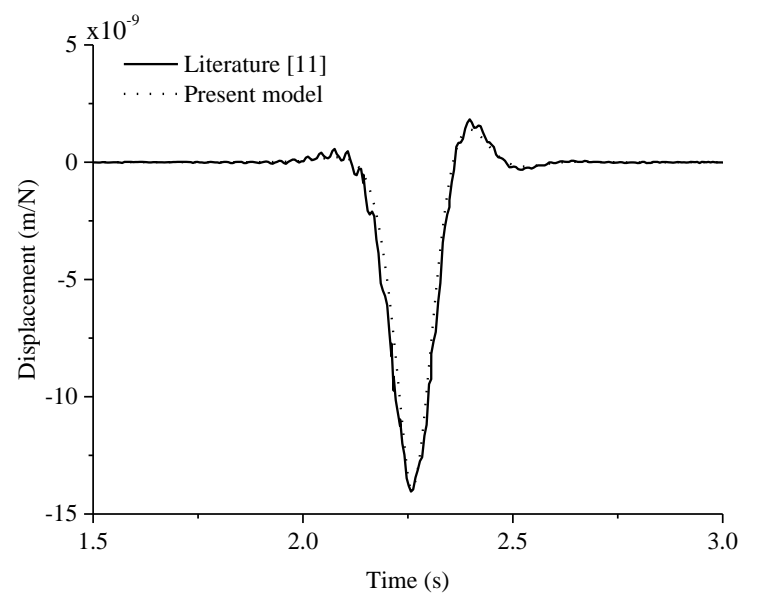

(b)

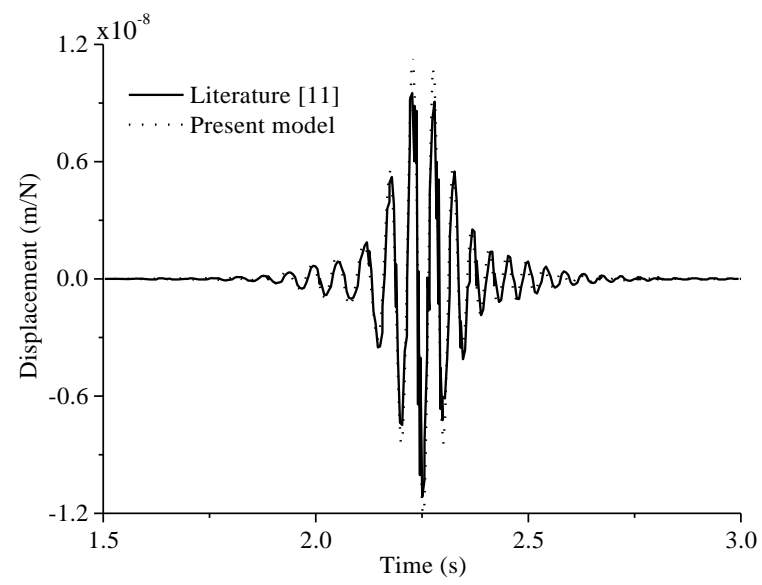

Fig. 5 Displacement at mid-span due to a harmonic moving load at (a) $0 \mathrm{~Hz}$ and (b) $20 \mathrm{~Hz}$

A second comparison is made with results from Reference [13]. This gave an analytical solution of response of a curved track continuously supported by a Winkler foundation under moving loads. Unlike [11], the results include the lateral and torsional response. The calculation parameters from [13] are shown in Table 2.

Table 2 Parameters of calculation in [13]

\begin{tabular}{ll}
\hline Young's modulus of rail $E$ & $2.0 \times 10^{11} \mathrm{~N} / \mathrm{m}^{2}$ \\
Shear modulus of rail $G$ & $8.33 \times 10^{10} \mathrm{~N} / \mathrm{m}^{2}$ \\
Cross-sectional area $A$ & $7.69 \times 10^{-3} \mathrm{~m}^{3}$ \\
Second moment of area of rail for vertical bending $I_{x}$ & $3.055 \times 10^{-5} \mathrm{~m}^{4}$ \\
Second moment of area of rail for lateral bending $I_{y}$ & $0.513 \times 10^{-5} \mathrm{~m}^{4}$ \\
Torsional constant of rail $I_{d}$ & $0.423 \times 10^{-5} \mathrm{~m}^{4}$ \\
Density of rail $\rho$ & $7800 \mathrm{~kg} / \mathrm{m}^{3}$ \\
Vertical stiffness of foundation $k_{v}$ & $10 \mathrm{MN} / \mathrm{m}$ \\
Vertical damping coefficient of foundation $c_{v}$ & $2.45 \mathrm{kNs} / \mathrm{m}$ \\
Lateral and axial stiffnesses of foundation, $k_{h}$ and $k_{z}$ & $5.5 \mathrm{MN} / \mathrm{m}$ \\
Lateral and axial damping coefficients of foundation, $c_{h}$ and $c_{z}$ & $1.82 \mathrm{kNs} / \mathrm{m}^{\prime}$ \\
Torsional stiffness of foundation $k_{r}$ & $71 \mathrm{kNm} / \mathrm{rad}$ \\
Torsional damping coefficient of foundation $c_{r}$ & $4.84 \mathrm{Nms} / \mathrm{rad}$ \\
Radius of curvature $R$ & $1000 \mathrm{~m}$ \\
Speed of load $v$ & $250 \mathrm{~km} / \mathrm{h}$ \\
\hline
\end{tabular}


The lateral, vertical and torsional displacements of the rail under a combination of moving loads of gravity and centrifugal forces are compared in Fig. 6. As can be seen from Fig. 6, excellent agreement is found for responses of the curved rail calculated by models in [13] and proposed in this paper.

(a)

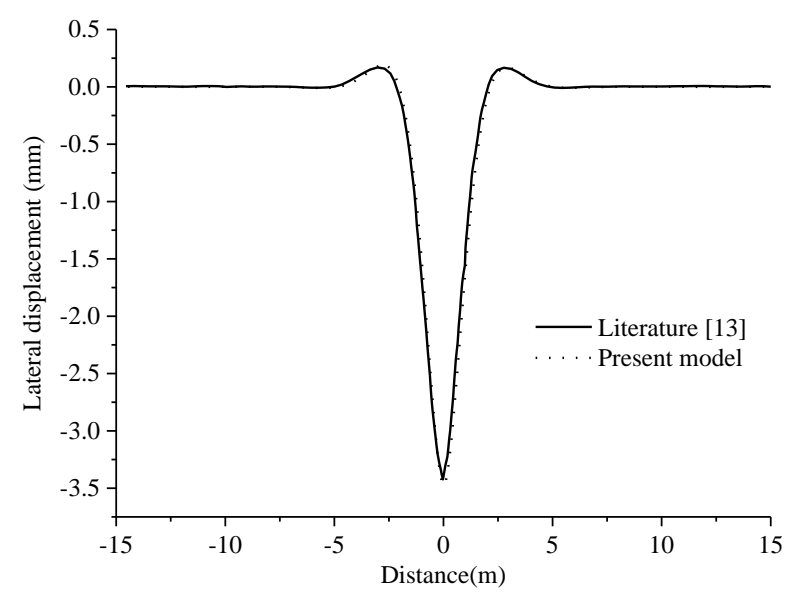

(b)

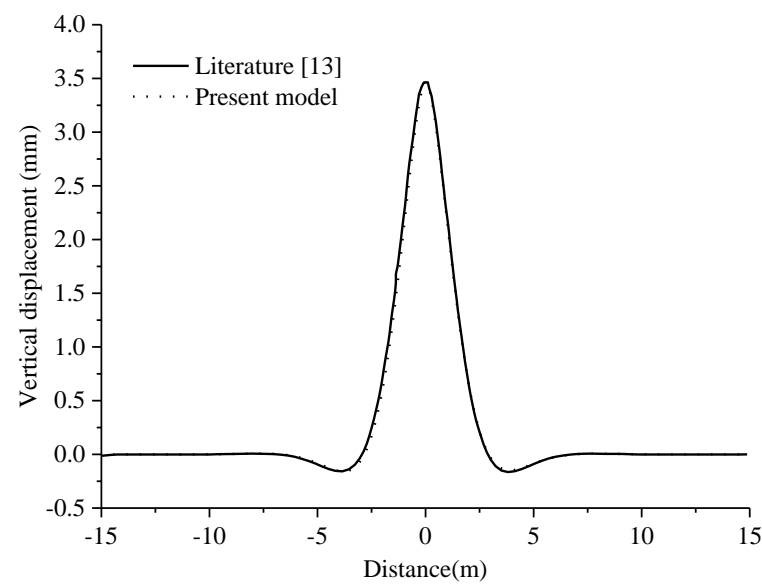

(c)

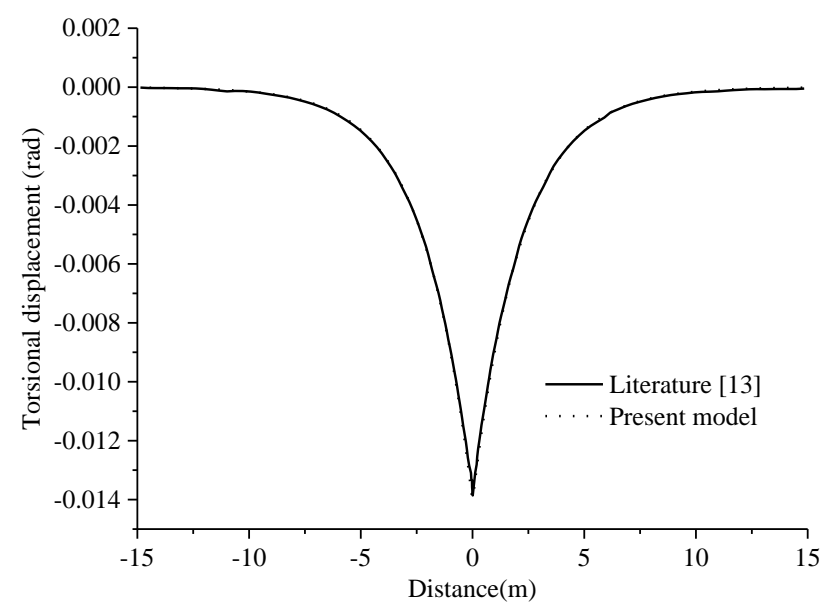

Fig. 6 (a) Lateral, (b) vertical and (c) torsional displacements of the rail under a moving load calculated by models in [13] and proposed in this paper

\section{Mobility and decay rate of the track due to non-moving harmonic loads}

In this section, the response of the track subjected to non-moving vertical and lateral harmonic unit forces is discussed. The response at the excitation point is expressed as the mobility, that is the velocity due to a unit harmonic force. In addition the decay rates of vibration along the track are 
determined. The vertical response at Position A and the lateral one at Position B on the rail head due to a vertical force applied at Point A or a lateral one at Point B (Fig. 3) are calculated. In this calculation, Point A is placed on the centreline of the rail head $(b=0)$. Parameters are chosen to correspond to a general non-ballast metro track. In this track, the GB60 rail and the DTVI 2 fastener are used. A fastener spacing of $0.6 \mathrm{~m}$ and a track radius of $300 \mathrm{~m}$ are considered in the following calculations. Unless otherwise stated the parameters are as listed in Table 3. The fastener is modelled as a single layer of springs and dashpots. The length of the reference cell is chosen as the fastener spacing, so a single fastener is included in the reference cell.

Table 3 Parameters of the metro track

\begin{tabular}{|c|c|}
\hline Young's modulus of rail $E$ & $2.059 \times 10^{11} \mathrm{~N} / \mathrm{m}^{2}$ \\
\hline Shear modulus of rail $G$ & $7.919 \times 10^{10} \mathrm{~N} / \mathrm{m}^{2}$ \\
\hline Cross-sectional area $A$ & $7.725 \times 10^{-3} \mathrm{~m}^{3}$ \\
\hline Second moment of area of rail for vertical bending $I_{x}$ & $3.217 \times 10^{-5} \mathrm{~m}^{4}$ \\
\hline Second moment of area of rail for lateral bending $I_{y}$ & $0.528 \times 10^{-5} \mathrm{~m}^{4}$ \\
\hline Torsional constant of rail $I_{d}$ & $0.215 \times 10^{-5} \mathrm{~m}^{4}$ \\
\hline Polar moment of inertia of rail $I_{0}$ & $3.714 \times 10^{-5} \mathrm{~m}^{4}$ \\
\hline Cross-sectional shear factor for vertical bending $K_{x}$ & 0.4507 \\
\hline Cross-sectional shear factor for lateral bending $K_{y}$ & 0.5329 \\
\hline Damping loss factor of rail $\eta$ & 0.01 \\
\hline Density of rail $\rho$ & $7850 \mathrm{~kg} / \mathrm{m}^{3}$ \\
\hline Vertical stiffness of fastener $k_{v}$ & $60 \mathrm{MN} / \mathrm{m}$ \\
\hline Vertical damping coefficient of fastener $c_{v}$ & $0.04 \mathrm{MNs} / \mathrm{m}$ \\
\hline Lateral and axial stiffnesses of fastener, $k_{h}$ and $k_{z}$ & $25 \mathrm{MN} / \mathrm{m}$ \\
\hline Lateral and axial damping coefficients of fastener, $c_{h}$ and $c_{z}$ & $16.7 \mathrm{kNs} / \mathrm{m}$ \\
\hline Torsional stiffness of fastener $k_{r}$ & $337.5 \mathrm{kNm} / \mathrm{rad}$ \\
\hline Torsional damping coefficient of fastener $c_{r}$ & $0.225 \mathrm{kNms} / \mathrm{rad}$ \\
\hline Fastener spacing $L$ & $0.6 \mathrm{~m}$ \\
\hline Radius of curvature $R$ & $300 \mathrm{~m}$ \\
\hline
\end{tabular}

If a vertical harmonic force is applied on the centreline of the rail head, only out-of-plane dynamic responses of the rail are obtained, which include vertical and torsional responses. However, the torsional response around the shear centre of the rail leads to a lateral response at the rail head. On the other hand, a lateral force applied on the rail head can be decomposed into a lateral force at 
the centroid and a torsional moment, so the dynamic response consists of both in-plane and out-of-plane responses according to Fig. 3.

The vertical and lateral point mobilities and the cross mobility at the rail head are shown in Fig. 7. In each case the mobility is shown at mid-span between two fasteners and directly above a fastener. From Fig. 7a, the vertical mobility has a peak at about $200 \mathrm{~Hz}$, which corresponds to the resonance of the rail mass on the vertical fastener stiffness. At about $1100 \mathrm{~Hz}$ there is a sharp peak at mid-span and a corresponding dip above a fastener, which is the pinned-pinned resonance ${ }^{3,18}$ at which half a bending wavelength fits within one sleeper span. Moreover, the mobility of the curved rail supported by a continuous elastic foundation is also shown. Compared with the result for discrete fasteners, the results from this model show the mean trend; the pinned-pinned resonance disappears for the continuous foundation, while the first peak has the same frequency. Fig. 7c shows the corresponding lateral mobility. This has a higher amplitude than the vertical mobility due to the smaller lateral fastener stiffness, lower bending stiffness and additional flexibility introduced by the torsion of the rail. The first peak in this case corresponds to the resonance of the rail mass on the lateral stiffness of the fastener. Two peaks are found at mid-span, at about 530 and $640 \mathrm{~Hz}$, caused by the pinned-pinned resonances of lateral and torsional motions of the rail. Again, corresponding dips are found above a fastener. Higher order pinned-pinned modes are seen at higher frequencies. The result for the continuously supported rail is again shown. Fig. $7 \mathrm{~b}$ shows the vertical/lateral cross mobility of the rail. The magnitude is much smaller than either vertical or lateral point mobilities. The first peak here occurs at a similar frequency to those found in the vertical and lateral mobility. Both the vertical and torsional pinned-pinned modes of the rail appear in the cross mobility. 
(a)

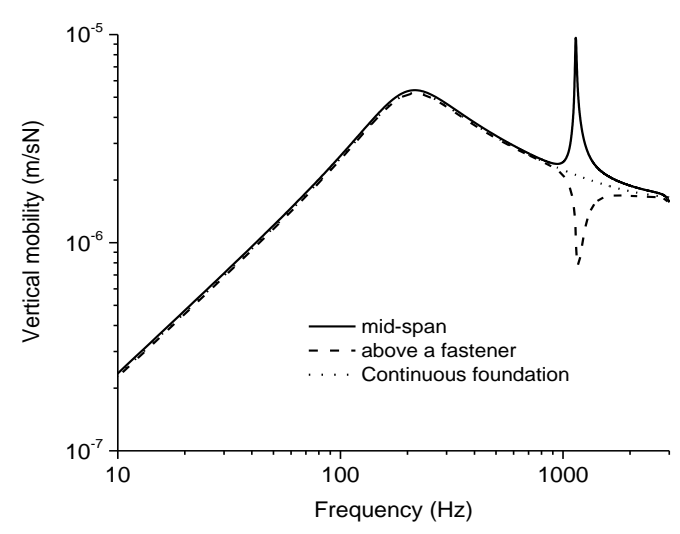

(c) (b)

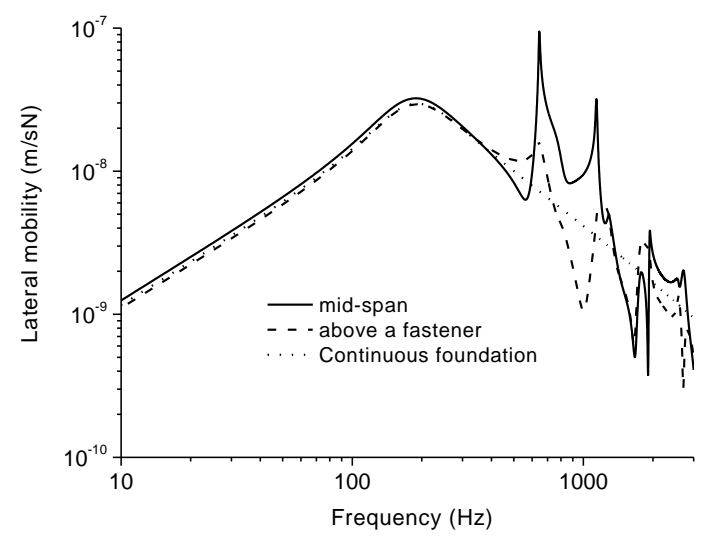

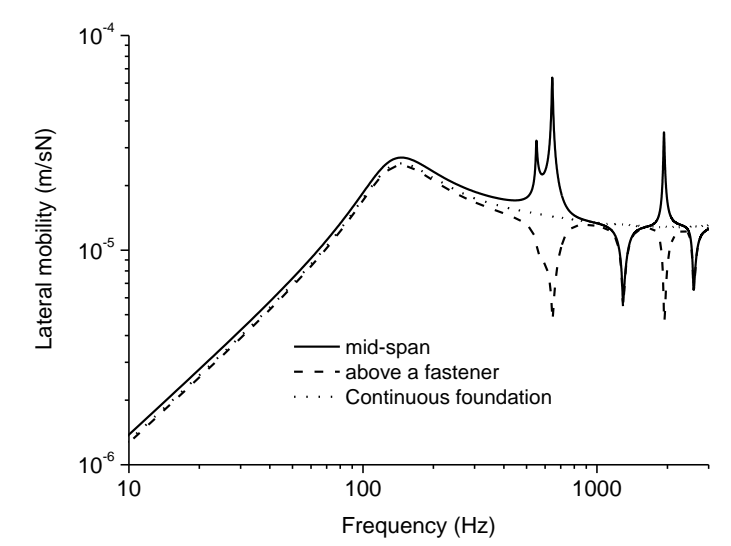

Fig.7 (a) Vertical and (b) lateral mobility at rail head under a vertical force, and (c) lateral mobility under a lateral force $(0.6 \mathrm{~m}$ fastener spacing, $300 \mathrm{~m}$ radius $)$

The effects on the frequency response of changes in the fastener stiffness, the fastener damping, the fastener spacing and the curvature of the track are next discussed.

The vertical and lateral (cross) mobility at the rail head, both above a fastener and at mid-span, due to a vertical force are shown for different values of vertical fastener stiffness in Fig. 8. From Fig. $8 \mathrm{a}$, the frequency of the first peak rises with increasing stiffness and the response amplitude at the peak becomes larger. However, the frequency and amplitude at the pinned-pined resonance are not affected. Fig. 8b shows the vertical/lateral cross mobility of the rail which shows similar trends.

The vertical and lateral responses at the rail head under a lateral force are shown for changes in lateral fastener stiffness in Fig. 9a and 9b. Similar trends are found to those seen in Fig. 8 for the effect of vertical stiffness. Again, the fastener stiffness has little influence on the pinned-pinned resonances of the rail. 
(a)

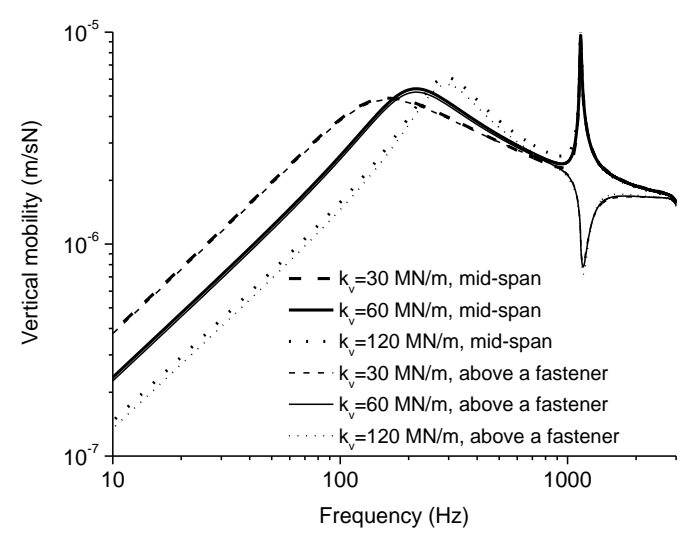

(b)

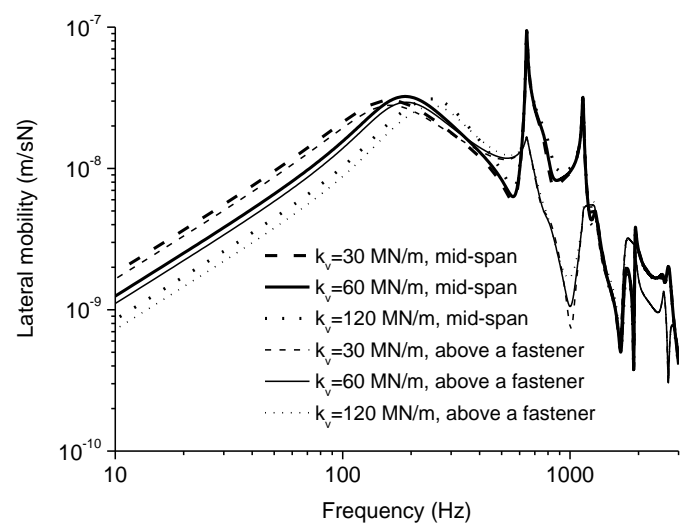

Fig.8 (a) Vertical and (b) lateral mobility (cross) at rail head under a vertical force, with changes of the vertical fastener stiffness ( $0.6 \mathrm{~m}$ fastener spacing, $300 \mathrm{~m}$ radius $)$

(a)

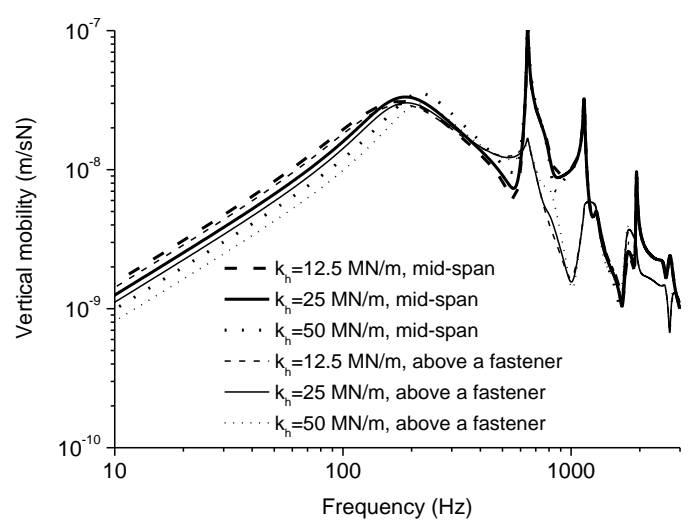

(b)

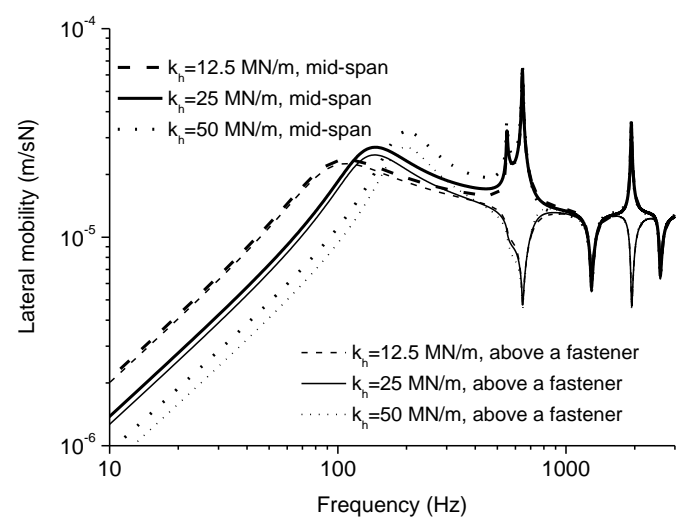

Fig.9 (a) Vertical (cross) and (b) lateral mobility at rail head under a lateral force, with changes of the lateral fastener stiffness ( $0.6 \mathrm{~m}$ fastener spacing, $300 \mathrm{~m}$ radius $)$

Fig. 10 shows the effect of changes in the fastener damping coefficient on the vertical and lateral mobility at the rail head. As the damping is increased, the amplitude of the mobility at the first peak is reduced. The pinned-pinned resonance frequencies become sharper with increasing damping as the fastener constrains the rail more. For low damping some oscillation can be seen in the mobility which is caused by the truncation of the number of fasteners. 
(a)

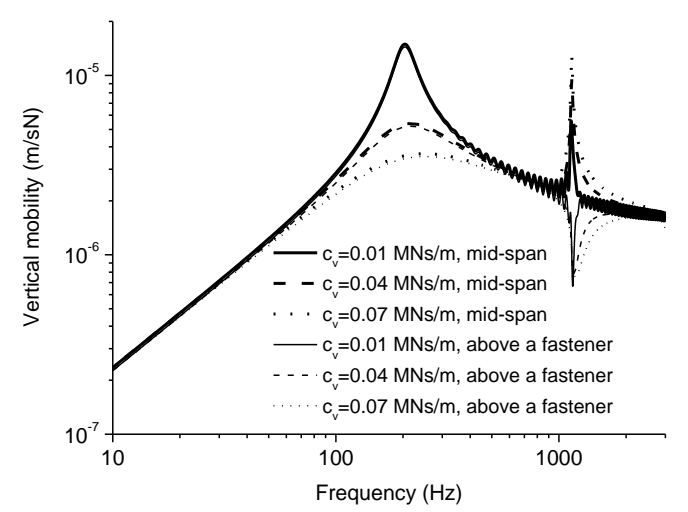

(b)

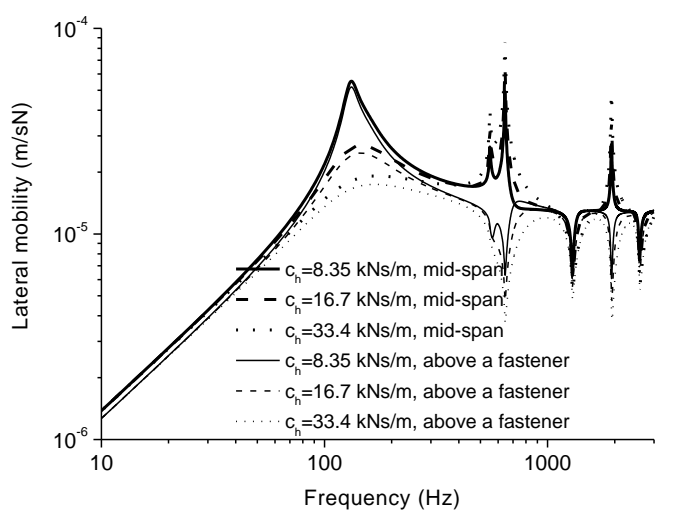

Fig.10 (a) Vertical mobility at rail head under a vertical force, and (b) lateral mobility under a lateral force, with changes of the fastener damping ( $0.6 \mathrm{~m}$ fastener spacing, $300 \mathrm{~m}$ radius)

The effect of changing the fastener spacing on the vertical and lateral point mobility of the rail is shown in Fig. 11. The fastener spacing affects the pinned-pinned frequencies significantly. These frequencies drop and the corresponding amplitudes rise as the spacing is increased. Moreover, as the fastener spacing is increased, the effective stiffness (per unit length) of the support is reduced so that the frequency of the first peak reduces and the mobility amplitude at the first peak increases.

(a)

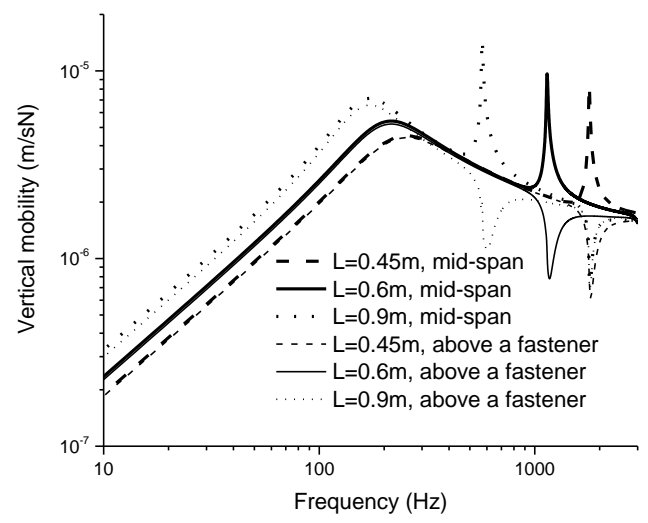

(b)

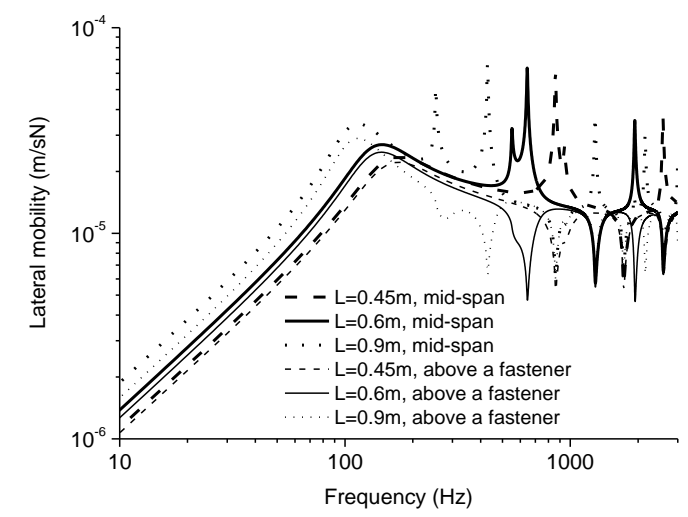

Fig.11 (a) Vertical mobility at rail head under a vertical force, (b) lateral mobility under a lateral force, with changes of the fastener spacing (300 m radius)

From the above results, which are all for a curved track with a radius $300 \mathrm{~m}$, it can be seen that the effects of changes to the stiffness, the damping and the spacing of the fasteners are similar to the effects seen for a straight track $^{26}$. 
The effect of changing the curvature on the mobility of the rail is shown in Figs 12 and 13. The radius of curvature has a negligible influence on the vertical and lateral mobility of the rail when the radius is larger than about $15 \mathrm{~m}$ and $30 \mathrm{~m}$, respectively. The result for a straight track is not shown but has almost the same mobility as the curved track with $300 \mathrm{~m}$ radius in Figs 12 and 13b. Additionally, the torsional pinned-pinned resonance appears in the vertical mobility at about $640 \mathrm{~Hz}$ in the case of very small radii (Fig. 12a and 12b). The radius of curves on metro or railway lines is usually at least $100 \mathrm{~m}$, in which case changes of the radius do not affect the mobility of the track. On tram tracks smaller radii are found but they are still in the region where the influence is negligible. For very small radii there are some changes, although such small radii are not found in practice. However, the cross mobility at the rail head is influenced greatly by the curvature (Fig. 13a). The amplitude of this mobility increases significantly as the radius is reduced. The cross mobility for the straight track is also shown in Fig. 13a. This is much lower than the results for a curved track. The lateral force at the rail head causes a moment about the $z$-axis (see Fig. 3). The vertical displacement and rotation of the rail are produced for the curved track, while only the rotation of the rail occurs for the straight track. Therefore the cross mobility has a different trend for the curved and straight tracks.

(a)

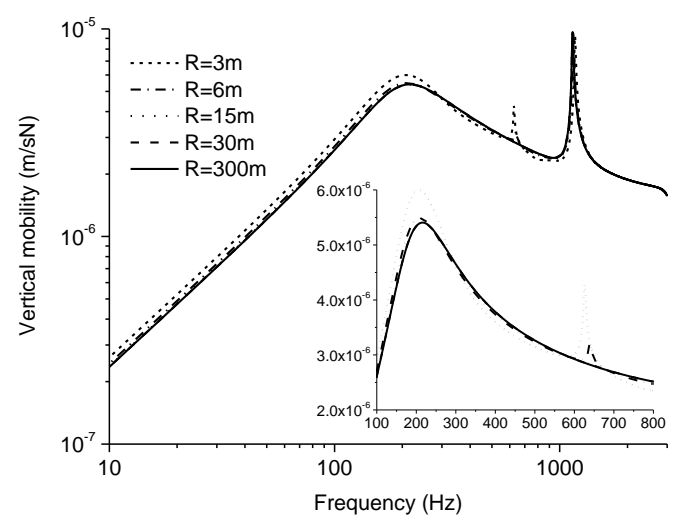

(b)

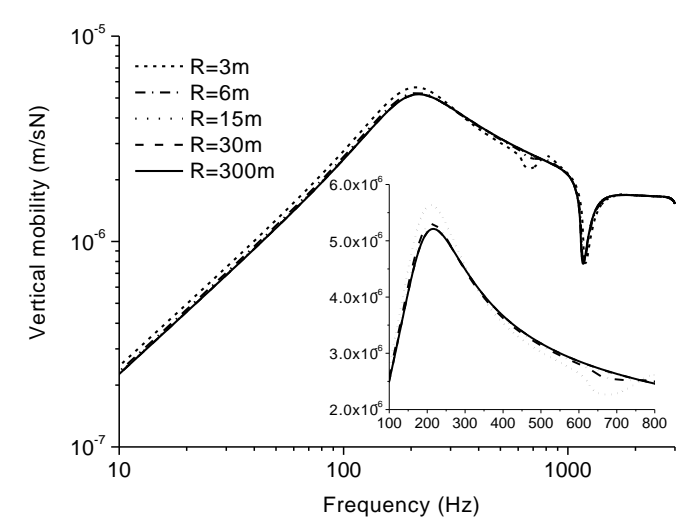

Fig.12 Vertical mobility at rail head at (a) mid-span and (b) above a fastener under a vertical force with changes of the curvature $(0.6 \mathrm{~m}$ fastener spacing) 
(a)

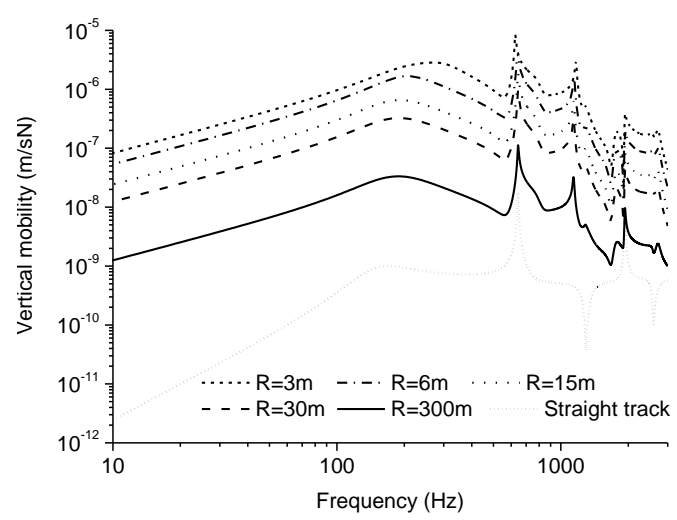

(b)

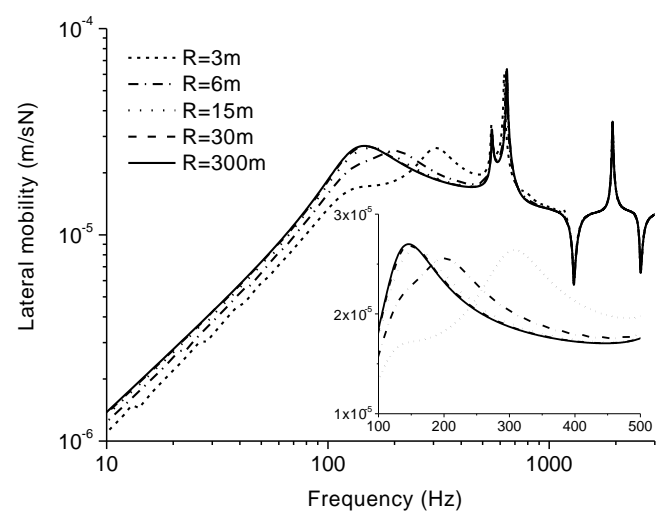

Fig. 13 (a) Vertical (cross) and (b) lateral mobility at rail head at mid-span under a lateral force with changes of the curvature $(0.6 \mathrm{~m}$ fastener spacing)

Fig. 14 shows the track decay rates obtained for different radii of curvature. The decay rate $(\mathrm{dB} / \mathrm{m})$ is evaluated here according to the standard $\mathrm{EN} 15461^{33}$ by determining the transfer mobility to positions along the rail due to a point force at mid-span. The decay rate is given by:

$$
D R \approx \frac{4.343}{\sum_{n=0}^{n_{\max }} \frac{\left|A\left(x_{n}\right)\right|^{2}}{\left|A\left(x_{0}\right)\right|^{2}} \Delta x_{n}}
$$

where $A\left(x_{n}\right)$ is the mobility at position $x_{n}$ along the track, $A\left(x_{0}\right)$ is the mobility at the excitation point $x_{0}$ at mid-span, and $\Delta x_{n}$ is the distance between $x_{n}$ and $x_{0}$. The curvature has an effect on the decay rates below $2000 \mathrm{~Hz}$ to some extent, while they are unaffected at high frequencies. The decay rates appear to increase as the radius reduces. There is a peak at about $640 \mathrm{~Hz}$ in the vertical decay rate due to the torsional pinned-pinned resonance of the rail for small radii. However, when the radius is greater than about $15 \mathrm{~m}$ it does not affect the vertical decay rate any more; similarly when the radius is greater than about $30 \mathrm{~m}$ it does not affect the lateral decay rate. These effects are mainly determined by the influence of the curvature on the point mobility, which appears in the equation for the decay rate (equation (44)). The track decay rates for a straight track, not shown, are virtually identical to those for a curved track with 300 m radius in Fig. 14. 
(a)

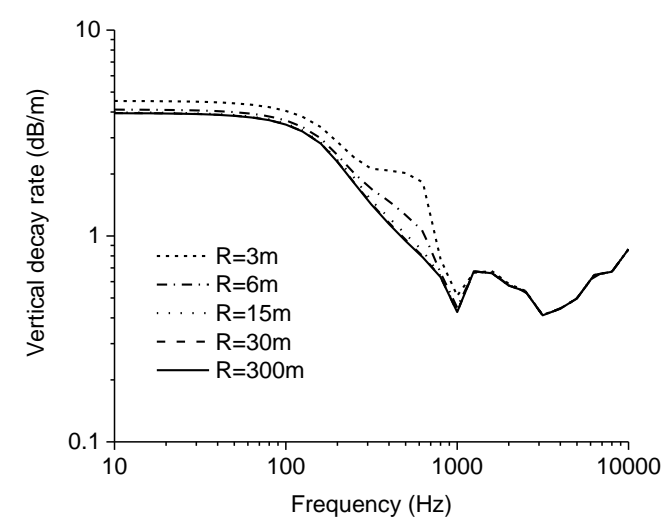

(b)

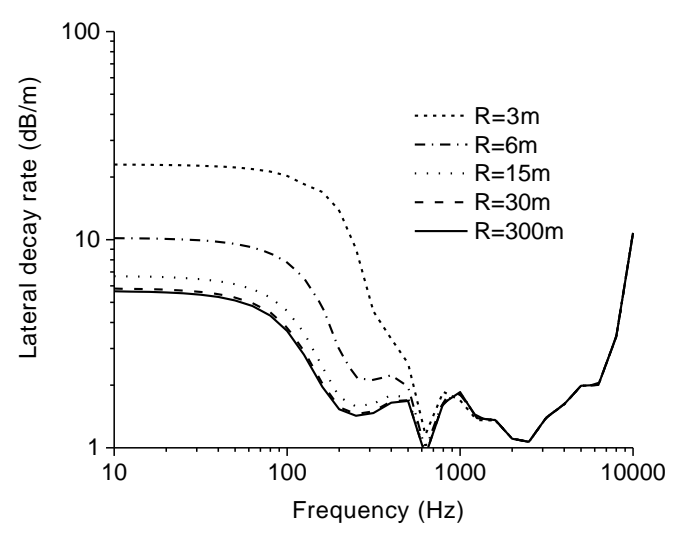

Fig. 14 (a) Vertical and (b) lateral decay rates with changes of the curvature ( $0.6 \mathrm{~m}$ fastener spacing)

\section{Velocity of the rail due to moving harmonic loads}

The vibration velocity of the rail subjected to moving vertical and lateral harmonic unit forces is discussed in this section. The vertical velocity at Position A and the lateral velocity at Position B under a vertical moving load at Point A or a lateral one at Point B (Fig. 3) are calculated. The vertical load moves along the centreline of the rail head. The parameters of the track used in these calculations are the same as in the previous section, as listed in Table 3. A fastener spacing of $0.6 \mathrm{~m}$ and a track radius of $300 \mathrm{~m}$ are considered. Unless otherwise stated the load speed is $100 \mathrm{~km} / \mathrm{h}$ and the excitation frequency is $200 \mathrm{~Hz}$, which is chosen to correspond to the peak in the vertical rail mobility.

Figs 15 and 16 show the frequency content of the velocity at the mid-span point on the rail for different load speeds 50 and $100 \mathrm{~km} / \mathrm{h}$. Fig 15 shows the results due to a vertical moving load and Fig. 16 shows the corresponding results for a lateral load. The frequency content of the response of a curved rail has similar characteristics to that of straight track under a moving harmonic load ${ }^{28}$. The rail has a relatively large dynamic response at and near the excitation frequency of the moving load, and the velocity away from this frequency attenuates quickly. There are two peaks, above and below the excitation frequency, due to the Doppler effect in the rail. For example, the frequencies of the two peaks are $193 \mathrm{~Hz}$ and $207 \mathrm{~Hz}$ for the load speed $100 \mathrm{~km} / \mathrm{h}$ in Fig. 15. The velocity in the vicinity of the excitation frequency drops as the load speed increases, while the width of the two peaks increases and the level rises at other frequencies. Moreover, some other small peaks can be 
found as well as the above-mentioned two large peaks. These small peaks are caused by the discrete fasteners.

(a)

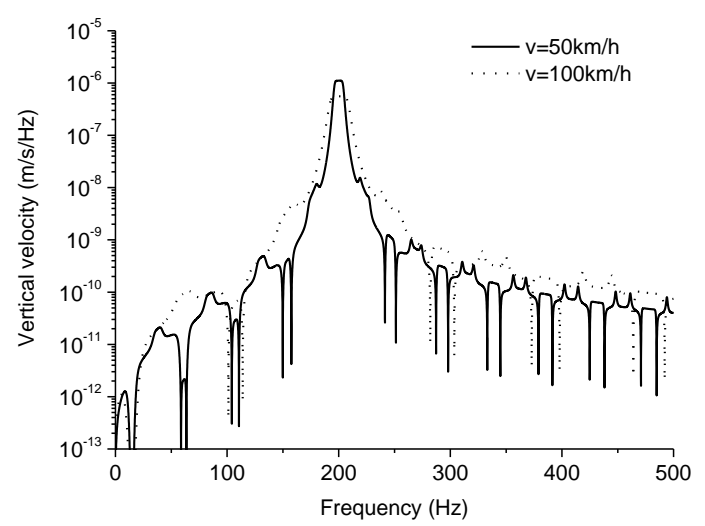

(b)

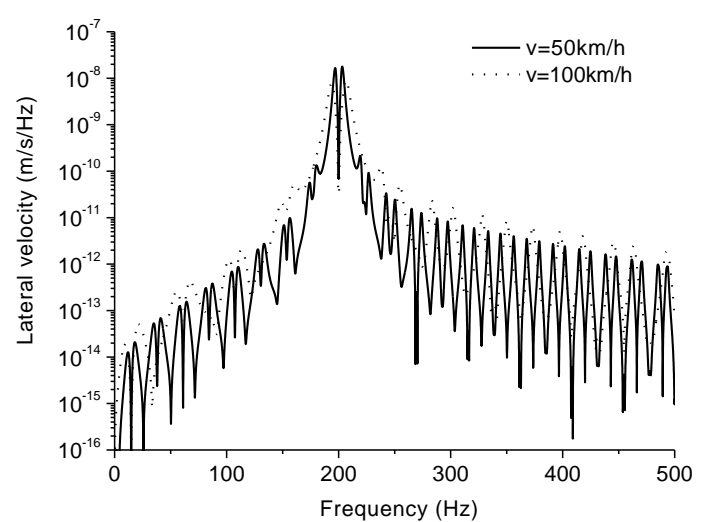

Fig.15 Frequency content of (a) vertical and (b) lateral velocity of rail under a vertical moving load at $200 \mathrm{~Hz}$, with changes of the load speed (0.6 m fastener spacing, $300 \mathrm{~m}$ radius)

(a)

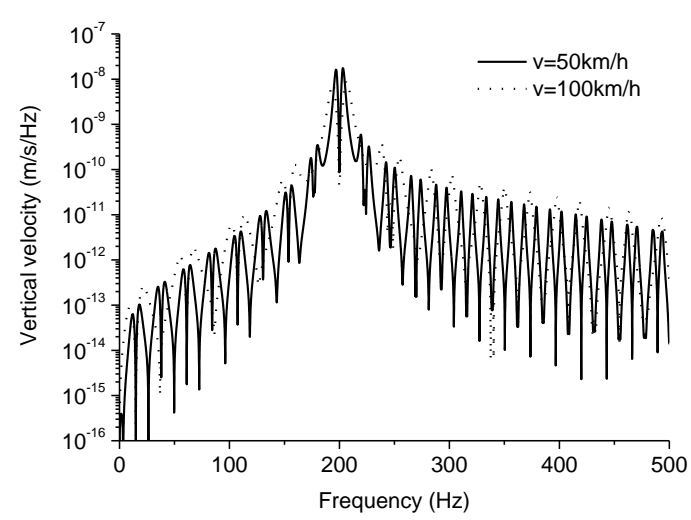

(b)

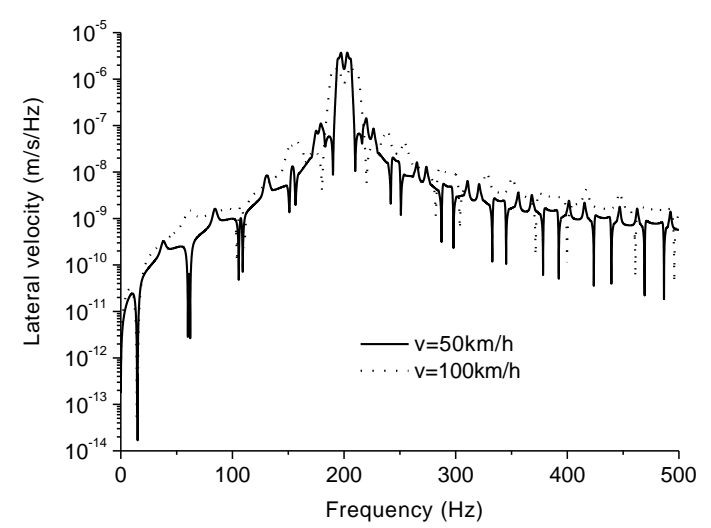

Fig.16 Frequency content of (a) vertical and (b) lateral velocity of rail under a lateral moving load at $200 \mathrm{~Hz}$, with changes of the load speed (0.6 $\mathrm{m}$ fastener spacing, $300 \mathrm{~m}$ radius)

The rail velocity obtained in the case of periodically-spaced discrete fasteners is compared with the result for a continuous foundation in Fig. 17. They have the similar dynamic behaviour at and near the excitation frequency. However the small peaks away from this frequency are not found for the continuous foundation, which confirms that they are the result of the discrete property of the fasteners. 
(a)

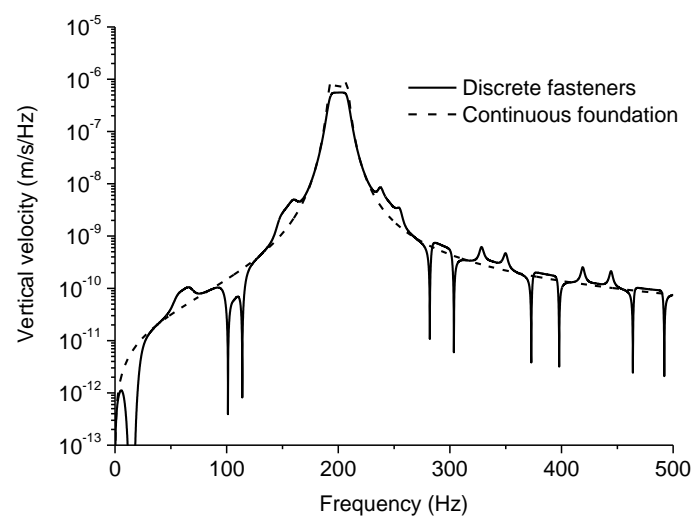

(b)

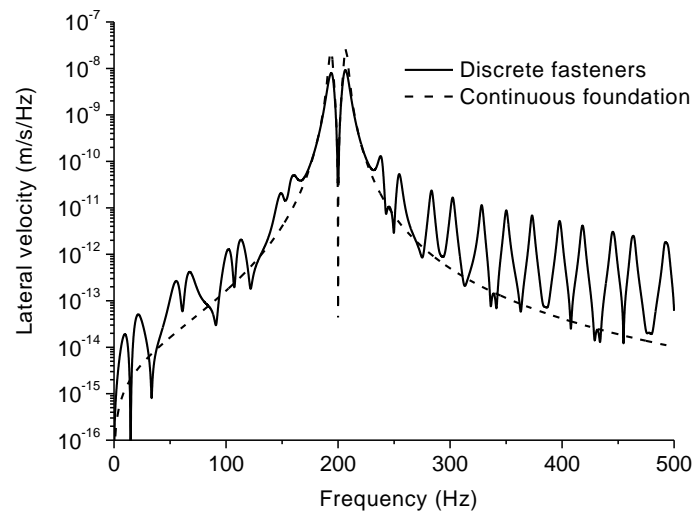

Fig. 17 Frequency content of (a) vertical and (b) lateral velocity of rail under a vertical moving load at $200 \mathrm{~Hz}$ in the cases of periodically discrete fasteners and the continuous foundation $(0.6 \mathrm{~m}$ fastener spacing, $300 \mathrm{~m}$ radius, $100 \mathrm{~km} / \mathrm{h}$ load speed)

To show the effect of different excitation frequencies, the maximum vertical and lateral velocity amplitudes obtained in the time domain for a vertical moving load are shown in Fig. 18 as a function of excitation frequency. It can be seen that these velocity amplitudes have a similar tendency to the mobility under the non-moving harmonic load (Fig. 7). However, as a result of the moving load, the peak at the pinned-pinned frequencies for the mid-span case is split into two peaks, whereas above a fastener the peaks and dips associated with the pinned-pinned frequencies that were found in the mobility disappear.

(a)

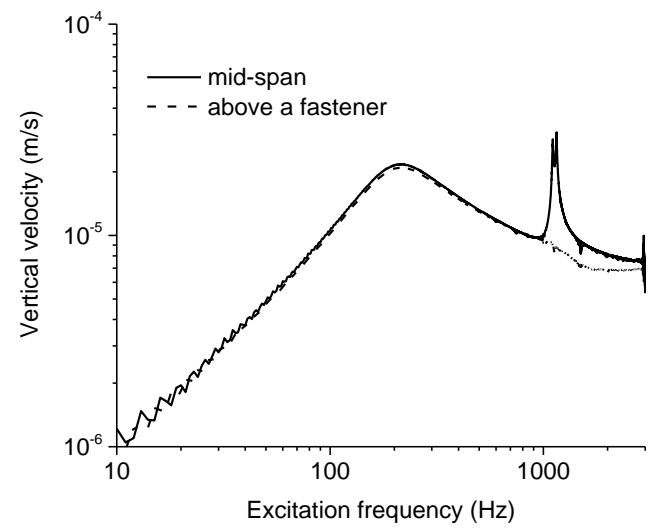

(b)

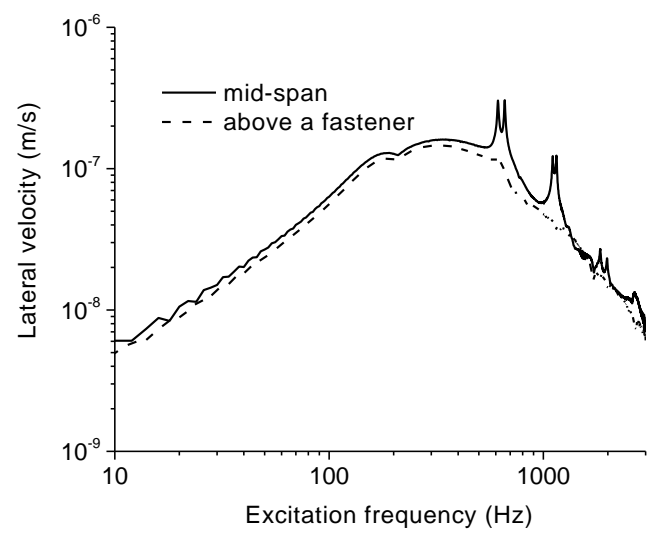

Fig. 18 Maximum of (a) vertical and (b) lateral rail velocity amplitude for different excitation frequencies under a vertical moving load in the time domain $(0.6 \mathrm{~m}$ fastener spacing, $300 \mathrm{~m}$ radius, $100 \mathrm{~km} / \mathrm{h}$ load speed) 
To see the effect if changing the radius of the curvature, the frequency content of the rail velocity for a moving vertical load at $200 \mathrm{~Hz}$ is shown in Fig. 19 for different values of the radius. Equivalent results for a moving lateral load are given in Fig. 20. The curvature has little influence on the vertical rail velocity for a vertical load (Fig. 19a) or the lateral rail velocity for a lateral load (Fig 20b). However, the lateral response to a vertical load (Fig. 19b) and vertical response to a lateral load (Fig. 20a) are greatly affected by the curvature. For a straight track, different from the vertical response to a lateral load, the lateral response to a vertical load is zero as the vertical load at the centreline of the rail head does not cause a moment about the $z$-axis.

(a)

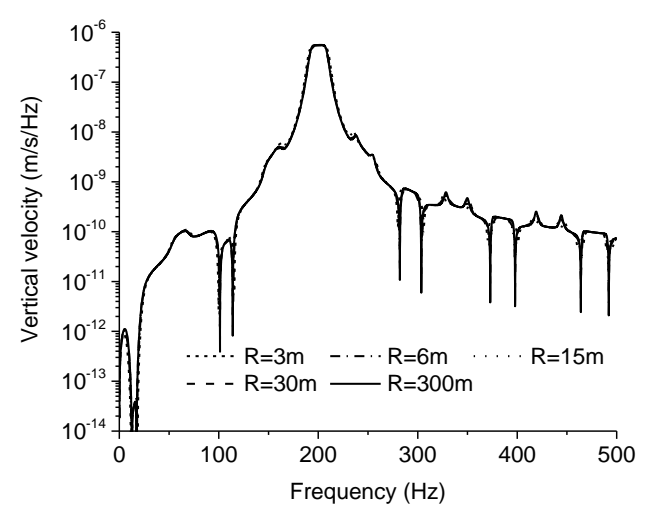

(b)

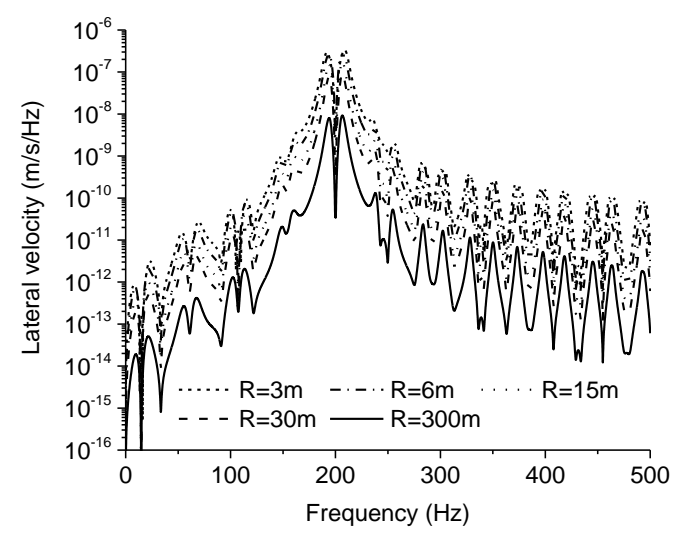

Fig. 19 Frequency content of (a) vertical and (b) lateral velocity of rail under a vertical moving load at $200 \mathrm{~Hz}$, with changes of the curvature $(0.6 \mathrm{~m}$ fastener spacing, $100 \mathrm{~km} / \mathrm{h}$ load speed $)$

(a)

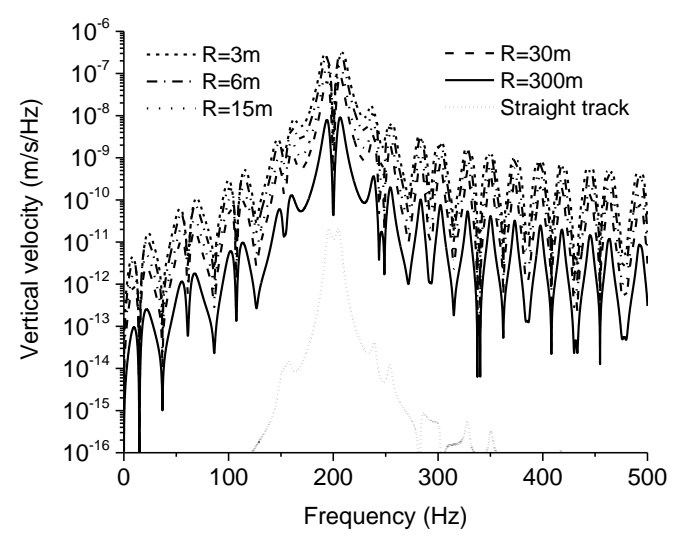

(b)

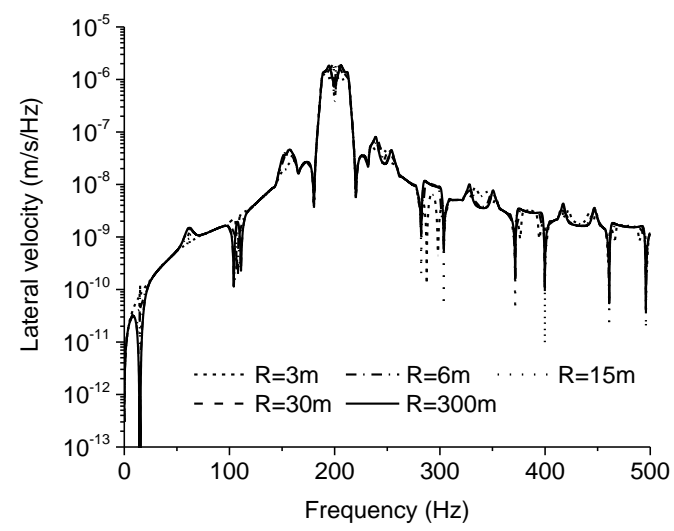

Fig. 20 Frequency content of (a) vertical and (b) lateral velocity of rail under a lateral moving load at $200 \mathrm{~Hz}$, with changes of the curvature (0.6 m fastener spacing, $100 \mathrm{~km} / \mathrm{h}$ load speed) 
Fig. 21 shows the maximum velocity amplitudes from the time domain at the mid-span position under a vertical and a lateral moving load, with an excitation frequency of $200 \mathrm{~Hz}$. These results are plotted against the radius of curvature. The maximum velocity amplitude becomes larger for small radii of curvature apart from the lateral response to a lateral load which reduces. The maximum velocity is greater for a larger fastener spacing due to the reduction in support stiffness per unit length of track. For the vertical velocity under the vertical load, the maximum response is independent of the curvature for radii greater than about $15 \mathrm{~m}$, while for the lateral velocity under the lateral load it is affected significantly by curvature for radii less than about $30 \mathrm{~m}$. The curvature has the same effect on the velocity of the rail as on the mobility due to a non-moving harmonic load (see Figs 12 and 13). When the radius of curvature is larger than $10 \mathrm{~m}$, the maximum vertical responses to a lateral force (and vice versa) are inversely proportional to the radius.

(a)

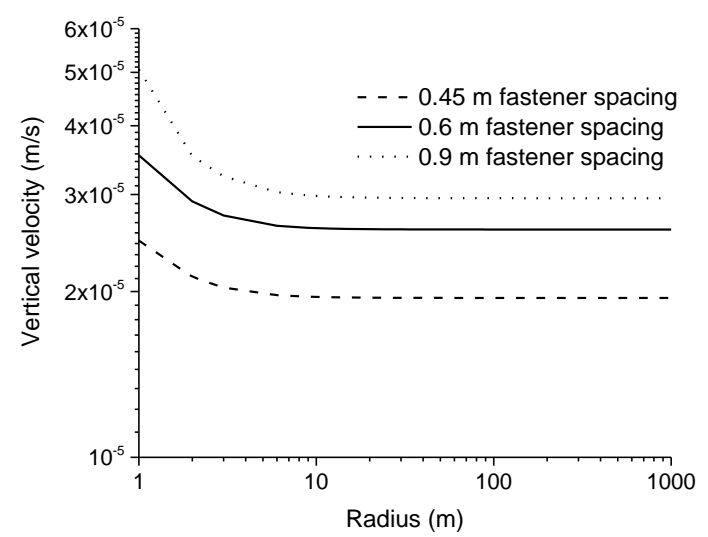

(c)

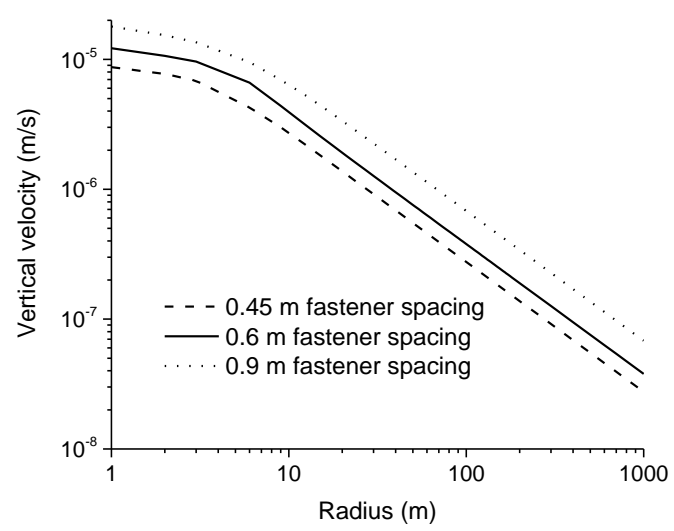

(b)

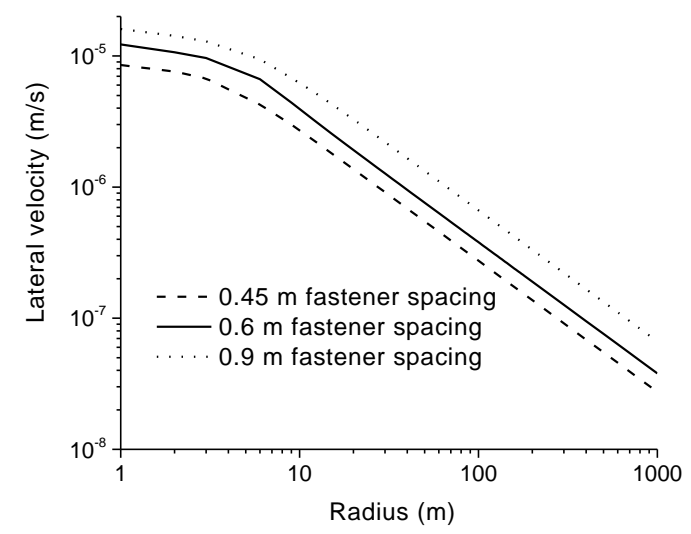

(d)

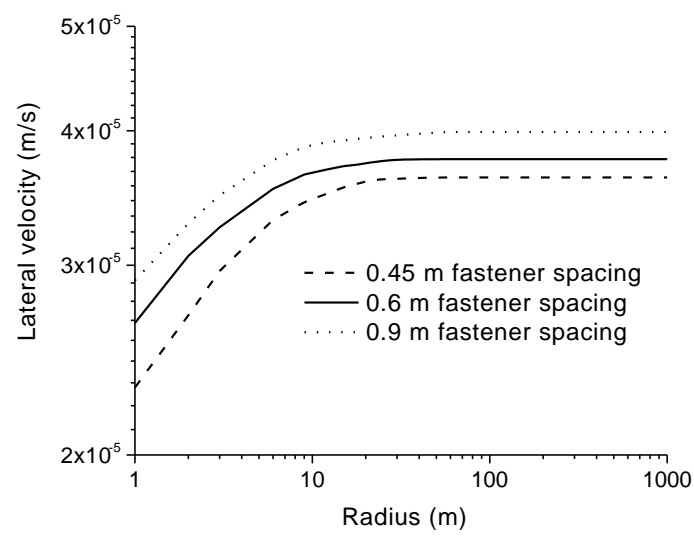

Fig. 21 Maximum of time history of (a) vertical and (b) lateral velocity amplitude of rail under a 
vertical moving load at $200 \mathrm{~Hz}$, and (c) vertical and (d) lateral velocity amplitude under a lateral moving load at $200 \mathrm{~Hz}$, with changes of the curvature (100 km/h load speed)

\section{Conclusions}

An analytical approach has been proposed to determine the response of a curved track subject to a non-moving or a moving harmonic load. The rail is modelled as a curved Timoshenko beam supported by periodically spaced discrete fasteners, and the dynamic responses in the vertical and lateral directions are taken into account. The displacement of the curved track in the frequency domain is expressed as the superposition of track modes which are associated with the Fourier series representation. The dynamic response of the curved track can be calculated efficiently using the periodic structure theory. The effect of various parameters on the dynamic behaviour of the track under non-moving and moving harmonic loads is discussed; these include the stiffness and damping of the fasteners, the fastener spacing, the radius of curvature, and the excitation speed.

The effects of varying the stiffness, damping and spacing of the fasteners on the dynamic response of the curved track with a large radius are similar to those found by previous authors for a straight track. However, when the radius of curvature is very small it has some influence on the dynamic behaviour of the track to some extent. Specifically, the radius significantly affects the vertical mobility of the curved rail when it is smaller than about $15 \mathrm{~m}$ and the lateral mobility when it is smaller than about $30 \mathrm{~m}$. Coupling between the vertical bending and torsion of the rail affects the vertical mobility for the curved track when the radius is less than about $15 \mathrm{~m}$. Moreover, the curvature has a significant influence on the vertical/lateral cross mobility, the magnitude of which increases as the radius becomes small. The curvature leads to coupling of vertical and torsional motions and of lateral and longitudinal motions.

The curvature has an effect on the track decay rate below $2000 \mathrm{~Hz}$. The decay rates increase as the radius is reduced for both vertical and lateral excitation. Because the track decay rate (according to the standard [33]) depends on the point mobility of rail, these effects are mainly determined by the influence of the curvature on the point mobility.

The frequency content of the dynamic response of the curved rail under moving harmonic loads has similar characteristics to those of a straight track. The curvature has little influence on the 
vertical or lateral velocities of the rail for forces in the corresponding direction, whereas it has significant effect on the vertical velocity due to a lateral force and vice versa. When the radius is larger than $10 \mathrm{~m}$, the maximum vertical/lateral cross amplitudes in the time domain are found to be inversely proportional to the radius. Furthermore, the amplitude of the vertical rail velocity for a vertical load is affected by the curvature when the radius is less than about $15 \mathrm{~m}$, and the lateral vibration under a lateral load is affected when the radius is less than about $30 \mathrm{~m}$.

\section{Declaration of Conflicting Interests}

The author(s) declared no potential conflicts of interest with respect to the research, authorship, and/or publication of this article.

\section{Funding}

The author(s) disclosed receipt of the following financial support for the research, authorship, and/or publication of this article: This work is part of a research project supported by National Natural Science Foundation of China (grant no. 51378001).

\section{References}

[1] Yuan Y, Liu WN, Liu WF. Propagation law of ground vibrations in the curve section of metro based on in-situ measurement. China Railway Science 2012; 33(4): 133-138.

[2] Knothe KL, Grassie SL. Modeling of railway track and vehicle/track interaction at high frequencies. Vehicle System Dynamics 1993; 22(3-4): 209-262.

[3] Thompson DJ. Railway noise and vibration: mechanisms, modelling and means of control. Oxford: Elsevier, 2009.

[4] Yang Y, Wu C and Yau J. Dynamic response of a horizontally curved beam subjected to vertical and horizontal moving loads. Journal of Sound and Vibration 2001; 242 (3): 519-537.

[5] Kang B, Riedel CH, Tan CA. Free vibration analysis of planar curved beams by wave propagation. Journal of Sound and Vibration 2003; 260: 19-44.

[6] Yu A, Yang J and Nie G. Analytical formulation and evaluation for free vibration of naturally curved and twisted beams. Journal of Sound and Vibration 2010; 329: 1376-1389.

[7]Washizu K. Some considerations on a naturally curved and twisted slender beam. Journal of 
Mathematical Physics1964; 43 (2): 111-116.

[8] Çalım F. Forced vibration of curved beams on two-parameter elastic foundation. Applied Mathematical Modelling 2012; 36: 964-973.

[9] Lee J. In-plane free vibration analysis of curved Timoshenko beams by the pseudospectral method. KSME International Journal2003; 17(8): 1156-1163.

[10] Howson WP and Jemah AK. Exact Out-of-Plane Natural Frequencies of Curved Timoshenko Beams. J. Eng. Mech.1999; 125(1): 19-25.

[11] Kostovasilis D, Koroma S, Hussein MFM, Owen J. A Comparison between the use of straight and curved beam elements for modelling curved railway tracks. 11th International Conference on Vibration Problems, Lisbon, Portugal, 2013.

[12] Kostovasilis D, Thompson DJ and Hussein MFM. The effect of vertical-lateral coupling of rails including initial curvature. 22nd International Congress on Sound and Vibration, Florence, Italy, 2015.

[13] Ang K and Dai J. Response analysis of a curved rail subject to a moving load. 11th International Conference on Vibration Problems, Lisbon, Portugal, 2013.

[14] Dai J and Ang K. Steady-state response of a curved beam on a viscously damped foundation subjected to a sequence of moving loads. Journal of Rail and Rapid Transit 2015; 229(4): 375-394.

[15] Li K, Liu W, Markine V and Han Z. Analytical study on the dynamic displacement response of a curved track subjected to moving loads. Journal of Zhejiang University-SCIENCE A 2013; 14(12): 867-879.

[16] Li K, Liu W, Markine V and Ma L. Analytical study on the vibration response of curved track subjected to moving load. 2nd International Conference on Railway Engineering, ICRE, Beijing, China, 2012, 556-562.

[17] Zhang H, Liu W, Li K and Su D. Analytical solution for dynamic response of curved rail subjected to moving train. Journal of Vibroengineering 2014; 16(4): 1392-8716.

[18] Grassie SL, Gregory R, Harrison D, Johnson K. The dynamic response of railway track to high frequency vertical excitation. Journal of Mechanical Engineering Science 1982; 24 (2): 77-90.

[19] Gry L and Gontier C. Dynamic modeling of railway track: a periodic model based on a generalized beam formulation. Journal of Sound and Vibration 1997; 199: 531-558.

[20] Sheng X and Li M. Propagation constants of railway tracks as a periodic structure. Journal of 
Sound and Vibration 2007; 299: 1114-1123.

[21] Degrande G, Clouteau D, et al. A numerical model for groundborne vibrations from underground railway traffic based on a periodic finite element-boundary element formulation. Journal of Sound and Vibration 2005; 293 (3-5): 645-666.

[22] Clouteau D, Arnst M, Al-Hussaini TM, Degrande G. Free field vibrations due to dynamic loading on a tunnel embedded in a stratified medium. Journal of Sound and Vibration 2005; 283(1-2): 173-199.

[23] Gupta S and Degrande G. Modelling of continuous and discontinuous floating slab tracks in a tunnel using a periodic approach. Journal of Sound and Vibration 2010; 329: 1101-1125.

[24] Gupta S, Liu WF, Degrande G, Lombaert G, Liu WN. Prediction of vibrations induced by underground railway traffic in Beijing. Journal of Sound and Vibration 2008; 310: 608-630.

[25] Chebli H, Clouteau D and Schmitt L. Dynamic response of high-speed ballasted railway tracks: 3D periodic model and in situ measurements. Soil Dynamics and Earthquake Engineering 2008; 28 : 118-131.

[26] Sheng X, Jones CJC and Thompson DJ. Responses of infinite periodic structures to moving or stationary harmonic loads. Journal of Sound and Vibration 2005; 282: 125-149.

[27] Ma L, Liu W and Li K. Fast Numerical Algorithm of Floating Slab Track Vibration Response under Moving Loads in the Frequency Domain. Journal of the China Railway Society 2014; 36(2): 86-94.

[28] Ma L. Study on the Model of Coupled Vehicle-Track and the Prediction Model for Tunnel-ground Vibration Response based on the Periodic-infinite Structure Theory. $\mathrm{PhD}$ thesis, Beijing Jiaotong University, 2014.

[29] Timoshenko SP. Vibration Problems in Engineering. D. Van Nostrand, New York, 1955.

[30]Belotserkovskiy PM. Forced oscillations of infinite periodic structures. Applications to railway track dynamics. Vehicle System Dynamics 1998; 29: 85-103.

[31] Belotserkovskiy PM. On the oscillations of infinite periodic beams subjected to a moving concentrated force. Journal of Sound and Vibration 1996; 193: 705-712.

[32] Wang T, Laskey AJ and Ahmad MF. Natural frequency for out-of-plane vibrations of continuous curved beams considering shear and rotary inertia. Journal of Solids Structures 1984; 20(3): 257-265. 
[33] Railway applications-Noise emission-Characterisation of the dynamic properties of track sections for pass by noise measurements (EN 15461: 2008+A1: 2010), European Standard EN 15461: 2008+A1: 2010, 2010. 